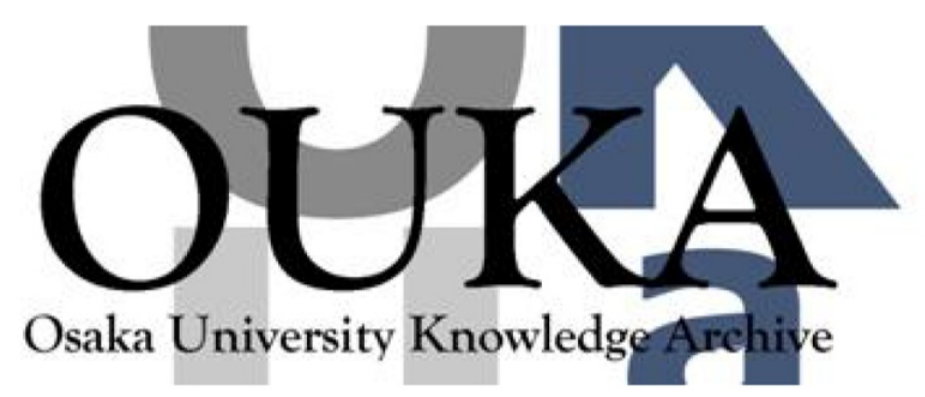

\begin{tabular}{|c|l|}
\hline Title & $\begin{array}{l}\text { Heat transfer in natural convection with } \\
\text { finite-sized particles considering thermal } \\
\text { conductance due to interparticle contacts }\end{array}$ \\
\hline Author(s) & $\begin{array}{l}\text { Takeuchi, Shintaro; Tsutsumi, Takaaki; } \\
\text { Kajishima, Takeo et al. }\end{array}$ \\
\hline Citation & $\begin{array}{l}\text { Computational Thermal Sciences. 7(5, 6) p. 385- } \\
\text { p. } 404\end{array}$ \\
\hline Issue Date & 2015 \\
\hline oaire:version & AM \\
\hline URL & https://hdl. handle. net/11094/57199 \\
\hline rights & \\
\hline Note & \\
\hline
\end{tabular}

Osaka University Knowledge Archive : OUKA

https://ir. Library. osaka-u. ac. jp/

Osaka University 


\title{
Heat transfer in natural convection with finite-sized particles considering thermal conductance due to inter-particle contacts
}

\author{
Shintaro Takeuchi ${ }^{1}$, Takaaki Tsutsumi ${ }^{1}$, Katsuya Kondo ${ }^{1}$, Takeshi Harada ${ }^{1}$ and \\ Takeo Kajishima ${ }^{1}$ \\ ${ }^{1}$ Department of Mechanical Engineering, Osaka University \\ 2-1 Yamada-oka, Suita-city, Osaka 565-0871 Japan
}

\begin{abstract}
Heat transfer problem in solid-dispersed two-phase flow is numerically studied. Temperature gradient within the finite-sized particles and inter-particle heat flux due to collisions are considered, and those effects on the flow structure and heat transfer are discussed. The interaction between fluid and particles is treated by our original immersed solid approach For the conjugate heat transfer problems, to satisfy the thermal condition at the fluid-solid interface, our interfacial heat flux model is employed. Also, the interfacial flux model is extended to incorporate the heat conduction due to inter-particle contacts, based on 2-D and axisymmetric contact heat resistance solutions. The method is applied to 2-D and 3-D natural convection problems including multiple particles in a confined domain under relatively low Rayleigh numbers $\left(10^{4} \sim 10^{6}\right)$. Heat transfer and particle behaviours are studied for different solid volume fractions (up to about $50 \%$ ) and heat conductivity ratios (solid to fluid) ranging between $10^{-3}$ and $10^{3}$. Under high solid volume fraction conditions, the particles are observed to form densely concentrated regions, where heat flow tends to channel through the contacting points. In three-dimensional solid-dispersed flows, by decomposing the heat flux into the contributions of the convection and conduction, the change of the major heat transfer mode is studied for different solid volume fractions and conductivity ratios.
\end{abstract}

keywords:

Multiphase flow, Solid-dispersion, Immersed solid object, Heat transfer, Thermal contact conduction 


\section{Introduction}

Solid-dispersed two-phase flow involving heat transfer is observed in industrial applications and natural phenomenon, where particle behaviour could be strongly influenced by the local temperature distribution and heat exchange between the solid particle and the surrounding fluid. For example, in catalyst engineering, a small particle in the vicinity of a large particle experiences significant effect of conduction [McKenna et al. 1999], and the catalyst particle could encounter a meltdown depending on its size and surrounding gas flow [McKenna et al. 1995]. As both continuum and dispersed components move in the practical particulate flows, it is essential to consider the heat convection by the respective components (i.e., fluid and individual particles) as well as the heat exchange at the interface. For heat transfer problems in two-phase flows with finite-sized particles, thermal conduction within the individual particles is of significance, and also, heat transfer through a contact surface of the particles is a non-negligible contribution in dense solid-dispersed flows.

For solving the heat and fluid flows around an object, extensive efforts have been paid for developing numerical algorithms for establishing the iso-temperature or iso-flux conditions at the fluid-solid interface. Some pioneering studies employ body-fitted mesh system around particle [Moukalled and Darwish 1997; Feng and Michaelides 2000] to facilitate the imposition of the interfacial condition, however, fixed grid approach is suited for solving the fluid flow around solid objects, particularly when a large number of relatively-moving particles are involved. Earlier studies worked out to implement Dirichlet and Neumann boundary conditions at the surface of a stationary object immersed in a fluid [Kim et al. 2001; Kim and Choi 2004; Pacheco et al. 2005; Pacheco-Vega et al. 2007; Ren et al. 2012]. However, in practical situations, the boundary condition is not either of those; rather, the temperature field around the fluid-solid interface is determined by the conductions in both phases and interfacial heat flux at the fluid-solid surface. Recently, this type of conjugate heat transfer was studied by Yu et al. [2006] in two-phase flow with freely-moving particles by their fictitious domain method with an extension of temperature distribution within the particles. They attempted to evaluate the convective and conductive contributions by particles on the heat transfer in the two-phase flow. This is one of the pioneering studies for dealing with the heat transfer in solid-dispersed two-phase flows with internal temperature distributions of the particles.

One of the present authors also proposed a method [Ueyama et al. 2011] for solving a heat-transfer problem in a fluid-solid two-phase flow on a fixed Cartesian mesh. Also, the present authors [Takeuchi et al. 2013; Tsutsumi et al. 2014] proposed a more concise interfacial heat flux model at the interface, and the method enables simulation of heat conduction within a moving particle and heat transfer in a flow including a number of finite-sized particles. The method has been applied to 2-D natural convection with particles, and an oscillatory motion of particles around the domain centre was reported. This was explained by the time 
difference for heat transfer through the fluid and solid phases, which highlights the importance of the temperature gradient within the particles. However, for both Yu et al. [2006] and the present authors [Takeuchi et al. 2013; Tsutsumi et al. 2014], the heat transfer models assume no heat exchange between the contacting particles, and the effect of the thermal contact conduction remains unexplored for finite-sized particles.

The fundamental concept of contact conduction is introduced with heat flux through a cross-section of contacting roughness elements, which is often modelled as heat conduction through constrictions of different sizes [Holm 1967]. In a heat exchange process of contacting objects, the surface temperatures are set to be different to allow a temperature gap at a small region of contact (contact site), and the apparent resistance for the heat flux is calculated as contact thermal resistance. Fenech and Rohsenow [1963] solved the equations of heat conduction for a contact-site model consisting of a constricted object surrounded by a fluid medium, and constructed an approximate solution of contact resistance in a suitable form to practical applications; the formula is applicable to the cases when the thermal conductivity of the medium is considerably smaller than that of metal, or when the actual contact area is extremely small compared with the apparent contact area. Yovanovich [1967] proposed a mathematical model for contact conduction through an elastically deformed sphere bounded by a pair of parallel plates, and derived an approximate analytical model in the axisymmetric system. Sanokawa [1968a,b,c,d] extensively studied thermal conductance through metallic contact with roughness, and by modelling the geometry of each contact site with a cylindrical constriction, he derived the rigorous solution of the thermal resistance and compared the calculated total resistance with the measurement [Sanokawa 1968a]. Other analytical models of thermal resistance for contacting solids immersed in a vacuum/fluid environment are reviewed by Madhusudana [1996].

Meanwhile, the attention also has been directed to thermal contact conduction in a granular bed with the effect of surrounding medium. Batchelor and O'Brien [1977] solved the inter-particle heat flux for contacting or nearly-contacting particles, and by applying the formulae to regularly/randomly-arranged stationary particles in a fluid, they studied the dependence of the solid-to-fluid conductivity ratio and interparticle gap on the effective conductivity of the granular bed. Sun and Chen [1988] showed 1-D theoretical and 2-D numerical analyses of contact conductance through the time-varying contact area during impact of two particles. They mentioned that, under typical conditions of heat flux problems in fluidized beds, heat exchange due to inter-particle collisions would not be a dominant mechanism, though, with highly conductive particles, the effect of the inter-particle heat exchange could be of importance in comparison to that of the conduction through the continuum phase. Vargas and McCarthy [2001] developed a granular heat transfer model by using a discrete element approach; a representative temperature is given to each pointparticle, and each particle in touch with particle $i$ exchanges heat with the same temperature of the particle $i$, assuming that the temperature at one contact point is not significantly different from that of another contact 
point. Vargas and McCarthy [2002] further included the effect of the heat transfer via the gas in the vicinity of the contact point in a packed bed of particles, and Sakurai et al. [2007, 2009] employed the developed model for heat transfer problem in a fluidized bed. However, as mentioned earlier about the characteristic effect of temperature gradient within finite-sized particles, the temperatures at contact points could vary and anisotropic arrangement could cause sequential heat conduction through aligned touching particles.

Despite the above fundamental studies on contact conduction, only a limited number of studies on the effect of inter-particle contact on the heat transfer in solid-dispersed two phase flows exists [Sakurai et al. 2007, 2009], in particular, the case of moving particles with internal temperature gradient is unexplored. Batchelor and O'Brien [1977] noted a sensitivity of the bulk conductivity of a granular material to a compression of the particles by an analysis of the heat flux through a contact circle between two spherical particles. Therefore, a thermal contact model needs account for the change of the contact heat resistance due to geometry of the contact site and the contact force.

The present study aims to develop an interfacial heat flux model in a two phase flow of finite-sized particles with a contact heat transfer between the particles. This is a first-step for developing a model of heat transfer in solid-dispersed two-phase flows. For solving the interaction between particle and fluid, our original immersed solid method [Kajishima and Takiguchi 2002; Yuki et al. 2007] is employed, which has been applied to a flow including a large number of particles [Kajishima et al. 2001; Kajishima and Takiguchi 2002; Kajishima 2004; Nishiura et al. 2006]. To take advantage of our immersed solid approach, the heat conduction problem in both fluid and solid phases is treated in an Eulerian way [Takeuchi et al. 2013; Tsutsumi et al. 2014]. The interfacial heat flux model is extended to include inter-particle heat conduction at contacting interface by incorporating a thermal contact model, based on the analytical models of contact heat resistance. The developed treatment of heat flux at the surface is shown to have good compatibility with our immersed solid approach. The method is applied to heat transfer problems in 2-D and 3-D particledispersed two-phase flows. And by changing the bulk solid volume fraction and the heat conductivity ratio (solid to fluid), characteristic heat transfer modes in particle-dispersed two-phase flows are studied. 


\section{Governing Equations and Numerical Methods}

\subsection{Governing equations}

An incompressible Newtonian fluid is assumed, and Boussinesq approximation is employed to include the effect of density fluctuation. The governing equations are the equations of continuity, momentum and energy:

$$
\begin{aligned}
& \nabla \cdot \boldsymbol{u}_{f}=0, \\
& \frac{\partial \boldsymbol{u}_{f}}{\partial t}+\boldsymbol{u}_{f} \cdot \nabla \boldsymbol{u}_{f}=-\frac{1}{\rho_{f}} \nabla p+\nu_{f} \nabla^{2} \boldsymbol{u}_{f}-\boldsymbol{g} \beta\left(T_{f}-T_{0}\right), \\
& \frac{\partial T_{f}}{\partial t}+\boldsymbol{u}_{f} \cdot \nabla T_{f}=\frac{1}{\rho_{f} c_{f}} \nabla \cdot\left(\lambda_{f} \nabla T_{f}\right),
\end{aligned}
$$

where $\boldsymbol{u}_{f}$ is the fluid velocity, $p$ the pressure, $\boldsymbol{g}$ the gravitational acceleration, $T_{f}$ the fluid temperature, $T_{0}$ the reference temperature, $c_{f}$ the specific heat and $\beta$ the thermal expansion coefficient. Density $\rho_{f}$, dynamic viscosity $\nu_{f}$ and thermal conductivity $\lambda_{f}$ are assumed to be constant. Viscous dissipation of kinetic energy is assumed to be negligible.

For solid objects, the conservation equations of translating and angular momenta for rigid body are as follows:

$$
\begin{aligned}
m_{s} \frac{\mathrm{d} \boldsymbol{v}_{s}}{\mathrm{~d} t} & =\boldsymbol{F}_{s}+\boldsymbol{G}_{s}, \\
\boldsymbol{I}_{s} \cdot \frac{\mathrm{d} \boldsymbol{\omega}_{s}}{\mathrm{~d} t} & =\boldsymbol{T}_{s}+\boldsymbol{N}_{s},
\end{aligned}
$$

where $m_{s}$ is the mass, $\boldsymbol{v}_{s}$ the translating velocity, $\boldsymbol{F}_{s}$ the hydrodynamic force, $\boldsymbol{G}_{s}$ the external force, $\boldsymbol{I}_{s}$ the inertia tensor, $\boldsymbol{\omega}_{s}$ the angular velocity, $\boldsymbol{T}_{s}$ the moment of the hydrodynamic force and $\boldsymbol{N}_{s}$ the external torque. The energy conservation for solid phase is treated in an Eulerian way:

$$
\frac{\partial T_{s}}{\partial t}+\boldsymbol{u}_{s} \cdot \nabla T_{s}=\frac{1}{\rho_{s} c_{s}} \nabla \cdot\left(\lambda_{s} \nabla T_{s}\right)
$$

where the subscript " $s$ " denotes the solid phase.

With the reference length $L$ and characteristic temperature difference $\Delta T$, the reference velocity is taken as $U=\sqrt{g \beta \Delta T L}$ and the reference pressure is $\rho_{f} U^{2}$, where $g=|\boldsymbol{g}|$. With those references, the governing equations for the fluid are non-dimensionalised with Rayleigh number (Ra) and Prandtl number (Pr):

$$
\mathrm{Ra}=\frac{g \beta \Delta T L^{3}}{\nu_{f} \lambda_{f} /\left(\rho_{f} c_{f}\right)}, \quad \operatorname{Pr}=\frac{\nu_{f}}{\lambda_{f} /\left(\rho_{f} c_{f}\right)} .
$$

In the following, as there is no possibility of misunderstandings, the non-dimensional variables are denoted with the same symbol as Eqs.(1)-(6). 


\subsection{Interaction between fluid and solid phases}

In the present study, the characteristic length of a solid particle is sufficiently resolved by the fluid meshes uniformly distributed over the computational domain, and the variables are arranged on the staggered points. At the fluid-solid interface, the computational cell is partially occupied by the solid object. Momentum exchange at the interfacial cells is solved by the immersed solid approach originally developed by Kajishima and the co-workers [Kajishima et al. 2001; Kajishima and Takiguchi 2002; Kajishima 2004]. This is briefly described below.

A velocity field $\boldsymbol{u}$ is established through volume-averaging the local fluid velocity $\boldsymbol{u}_{f}$ and the local particle velocity $\boldsymbol{u}_{s}$ in a cell:

$$
\boldsymbol{u}=(1-\alpha) \boldsymbol{u}_{f}+\alpha \boldsymbol{u}_{s},
$$

where $\alpha(0 \leq \alpha \leq 1)$ is the local solid volume fraction in the cell. The solid velocity $\boldsymbol{u}_{s}$ is decomposed into translating and rotating components as $\boldsymbol{u}_{s}=\boldsymbol{v}_{s}+\boldsymbol{\omega}_{s} \times \boldsymbol{r}_{s}$, where $\boldsymbol{r}_{s}$ is a position vector from the gravity centre. This mixture velocity field $\boldsymbol{u}$ is assumed to obey the following equation:

$$
\frac{\partial \boldsymbol{u}}{\partial t}=-\nabla p-(\boldsymbol{u} \cdot \nabla) \boldsymbol{u}+\sqrt{\frac{\mathrm{Pr}}{\mathrm{Ra}}} \nabla^{2} \boldsymbol{u}-\left(T-T_{0}\right) \boldsymbol{j}+\boldsymbol{f}_{s},
$$

where $\boldsymbol{j}$ is the unit vector in the vertical downward direction $(\boldsymbol{g} /|\boldsymbol{g}|)$, and $\boldsymbol{f}_{s}$ represents the interaction term which works to assign the mixture velocity to satisfy the no-slip condition at the interface [Kajishima et al. 2001; Kajishima and Takiguchi 2002; Kajishima 2004]. For spatial discretisation of Eq.(8), second-order finite difference schemes are used. For time-update, the 2nd-order Adams-Bashforth and Crank-Nicolson methods are employed for the convective and viscous terms, respectively. The pressure gradient term in Eq.(8) is treated implicitly by a fractional step method. With the time-updated velocity field, the fluid-solid interaction term $\boldsymbol{f}_{s}$ is modelled as

$$
\boldsymbol{f}_{s}^{n}=\frac{\alpha^{n}\left(\boldsymbol{u}_{s}^{n}-\boldsymbol{u}_{f}^{n+1}\right)}{\Delta t}
$$

where $\Delta t$ is the time increment. The energy equation is time updated by the 2 nd-order Adams-Bashforth method for the convective term and the Crank-Nicolson method for the diffusion term.

For motion of the particles, the force Eq.(9) is applied to the fraction of the solid in the interface cell with the opposite sign. The surface integration of the hydrodynamic forces is changed to the integration of $\boldsymbol{f}_{s}$ over the volume of the particle $V_{s}$ :

$$
\begin{aligned}
& \frac{\boldsymbol{v}_{s}^{n+1}-\boldsymbol{v}_{s}^{n}}{\Delta t}=m_{s}^{-1} \int_{V_{s}}\left(-\rho_{f} \boldsymbol{f}_{s}\right) \mathrm{d} V+m_{s}^{-1} \boldsymbol{G}_{s} \\
& \frac{\boldsymbol{\omega}_{s}^{n+1}-\boldsymbol{\omega}_{s}^{n}}{\Delta t}=\boldsymbol{I}_{s}^{-1} \cdot \int_{V_{s}} \boldsymbol{r}_{s} \times\left(-\rho_{f} \boldsymbol{f}_{s}\right) \mathrm{d} V+\boldsymbol{I}_{s}^{-1} \cdot \boldsymbol{N}_{s}
\end{aligned}
$$


where the superscripts represent time levels.

In the present study, the time integrations of the translating and angular momenta are carried out by an explicit multi-staged predictor and corrector method [Ueyama et al. 2011], which is briefly explained in the following. Note that the following algorithm is shown with the forward Euler method for the purpose of simplicity. However, higher order schemes are readily available. The prediction stage for Eqs.(4) and (5) is as follows:

$$
\begin{aligned}
\widetilde{\boldsymbol{v}}_{s} & =\boldsymbol{v}_{s}^{n}+\Delta t m_{s}^{-1} \boldsymbol{F}_{s}^{n}, \\
\widetilde{\boldsymbol{\omega}}_{s} & =\boldsymbol{\omega}_{s}^{n}+\Delta t \boldsymbol{I}_{s}^{-1} \cdot \boldsymbol{T}_{s}^{n},
\end{aligned}
$$

where $\sim$ stands for the predicted value, and

$$
\boldsymbol{F}_{s}^{n}=\int_{V_{s}^{n}}\left(-\rho_{f} \boldsymbol{f}_{s}^{n}\right) \mathrm{d} V, \quad \boldsymbol{T}_{s}^{n}=\int_{V_{s}^{n}} \boldsymbol{r}_{s} \times\left(-\rho_{f} \boldsymbol{f}_{s}^{n}\right) \mathrm{d} V .
$$

Then the solid velocities are updated as:

$$
\widetilde{\boldsymbol{u}}_{s}=\widetilde{\boldsymbol{v}}_{s}+\widetilde{\boldsymbol{\omega}}_{s} \times \boldsymbol{r}_{s}
$$

Based on the above translating and angular velocities, the displacement of the gravity centre $\boldsymbol{x}_{s}$ and the rotation angle $\boldsymbol{\theta}_{s}$ are obtained by the Crank-Nicolson method:

$$
\begin{aligned}
& \widetilde{\boldsymbol{x}}_{s}=\boldsymbol{x}_{s}^{n}+\frac{\Delta t}{2}\left(\widetilde{\boldsymbol{v}}_{s}+\boldsymbol{v}_{s}^{n}\right), \\
& \widetilde{\boldsymbol{\theta}}_{s}=\boldsymbol{\theta}_{s}^{n}+\frac{\Delta t}{2}\left(\widetilde{\boldsymbol{\omega}}_{s}+\boldsymbol{\omega}_{s}^{n}\right) .
\end{aligned}
$$

With the predicted position of the solid objects, the solid volume fraction at each computational cell is updated to obtain $\widetilde{\alpha}$ and the corresponding interaction term $\widetilde{\boldsymbol{f}}_{s}$. In the correction stage, $\widetilde{\boldsymbol{f}}_{s}$ and its first-order moment are summed over the cells occupied by the solid objects to update the solid translating and angular velocities:

$$
\begin{aligned}
\boldsymbol{v}_{s}^{n+1} & =\boldsymbol{v}_{s}^{n}+\frac{\Delta t}{2} m_{s}^{-1}\left(\boldsymbol{F}^{n}+\widetilde{\boldsymbol{F}}_{s}\right) \\
\boldsymbol{\omega}_{s}^{n+1} & =\boldsymbol{\omega}_{s}^{n}+\frac{\Delta t}{2} \boldsymbol{I}_{s}^{-1} \cdot\left(\boldsymbol{T}^{n}+\widetilde{\boldsymbol{T}}_{s}\right)
\end{aligned}
$$

where

$$
\widetilde{\boldsymbol{F}}_{s}=\int_{\widetilde{V}_{s}}\left(-\rho_{f} \widetilde{\boldsymbol{f}}_{s}\right) \mathrm{d} V, \quad \widetilde{\boldsymbol{T}}_{s}=\int_{\widetilde{V}_{s}} \boldsymbol{r}_{s} \times\left(-\rho_{f} \widetilde{\boldsymbol{f}}_{s}\right) \mathrm{d} V
$$

The solid velocity field at the next time step is obtained as:

$$
\boldsymbol{u}_{s}^{n+1}=\boldsymbol{v}_{s}^{n+1}+\boldsymbol{\omega}_{s}^{n+1} \times \boldsymbol{r}_{s}
$$




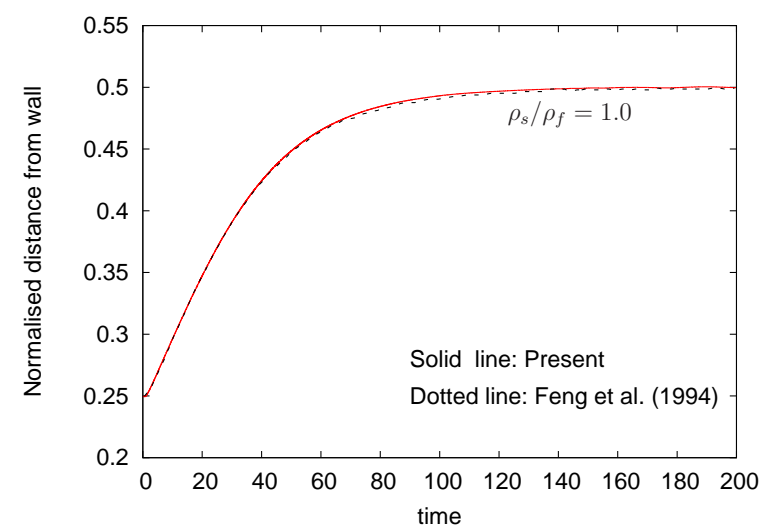

Figure 1: Time history of particle migration in a wall-driven shear flow. Neutrally-buoyant particle is employed. The bulk Reynolds number is fixed to 40. The result is compared with a reference numerical result (dotted line) by Feng et al. [1994].

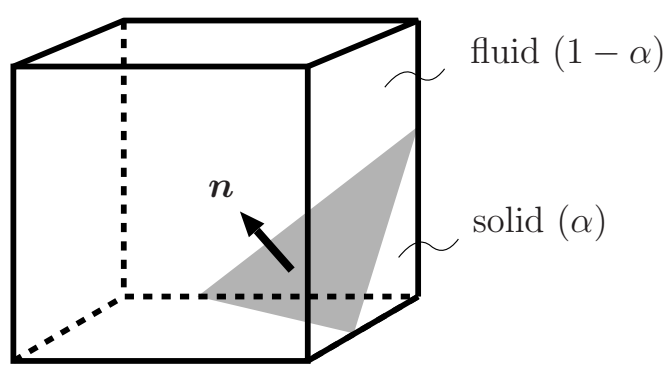

Figure 2: Schematic of the interfacial cell partially occupied by a solid object (solid volume fraction $\alpha$ ).

Finally, with the values in Eq. (15), the time-update of the position and rotation angles, $\boldsymbol{x}_{s}$ and $\boldsymbol{\theta}_{s}$, are completed by the Crank-Nicolson method:

$$
\begin{aligned}
& \boldsymbol{x}_{s}^{n+1}=\boldsymbol{x}_{s}^{n}+\frac{\Delta t}{2}\left(\boldsymbol{v}_{s}^{n+1}+\boldsymbol{v}_{s}^{n}\right) \\
& \boldsymbol{\theta}_{s}^{n+1}=\boldsymbol{\theta}_{s}^{n}+\frac{\Delta t}{2}\left(\boldsymbol{\omega}_{s}^{n+1}+\boldsymbol{\omega}_{s}^{n}\right) .
\end{aligned}
$$

The mixture velocity is also updated to keep the consistency with the above momentum exchange procedure:

$$
\boldsymbol{u}^{n+1}=\boldsymbol{u}_{f}^{n+1}+\frac{\Delta t}{2}\left(\boldsymbol{f}_{s}^{n}+\widetilde{\boldsymbol{f}}_{s}\right)
$$

This two-staged time-update method is shown to be applicable to fluid-solid interaction problems for neutrally-buoyant particle. Figure 1 shows a time history of particle migration in a wall-driven shear flow in a 2-D channel of width $L$. The result is compared with the available numerical result in the literature [Feng et al. 1994]. The particle diameter is $L / 4$, and the bulk Reynolds number and Froude number are 40 and 392, respectively. Initially, the particle is placed $L / 4$ away from a wall with zero velocity (i.e., with a finite velocity gap against the background shear flow). The graph shows that the present numerical result shows good agreement with the reference data. 


\subsection{Interfacial heat flux model}

Figure 2 is a schematic of an interfacial cell where a fraction of the solid object occupies the volume. The volume fraction in the cell is denoted as $\alpha$. The unit vector $\boldsymbol{n}$ represents the outward surface normal direction. The interfacial heat flux on the discretised field is summarised in the following.

In the interfacial cells (cell indices $i, j$ and $k$ ), the discretised temperature gradient $\left(\left.\nabla T\right|_{i j k}\right)$ is decomposed into the normal direction:

$$
\left(\left.\boldsymbol{n} \cdot \nabla T\right|_{i j k}\right) \boldsymbol{n}
$$

and tangential direction:

$$
\left.\nabla T\right|_{i j k}-\left(\left.\boldsymbol{n} \cdot \nabla T\right|_{i j k}\right) \boldsymbol{n}
$$

By multiplying the following averaged conductivities of the materials:

$$
\begin{aligned}
\frac{1}{\lambda_{h}} & =\frac{1-\alpha}{\lambda_{f}}+\frac{\alpha}{\lambda_{s}}, \\
\lambda_{a} & =(1-\alpha) \lambda_{f}+\alpha \lambda_{s},
\end{aligned}
$$

the heat flux in the respective directions are constructed, and the heat flux of the interfacial cell $\boldsymbol{q}_{\mathrm{I}}$ is given as the sum of those:

$$
-\boldsymbol{q}_{\mathrm{I}}=\left.\lambda_{h}(\boldsymbol{n n})_{i j k} \cdot \nabla T\right|_{i j k}+\left.\lambda_{a}(\boldsymbol{I}-\boldsymbol{n n})_{i j k} \cdot \nabla T\right|_{i j k} \cdot
$$

Therefore, the interfacial heat flux at the $(i, j, k)$ cell is computed by

$$
\boldsymbol{q}_{\mathrm{I}}=-\left(\begin{array}{ccc}
\lambda_{a}+\Delta \lambda n_{x}^{2} & \Delta \lambda n_{x} n_{y} & \Delta \lambda n_{x} n_{z} \\
\Delta \lambda n_{y} n_{x} & \lambda_{a}+\Delta \lambda n_{y}^{2} & \Delta \lambda n_{y} n_{z} \\
\Delta \lambda n_{z} n_{x} & \Delta \lambda n_{z} n_{y} & \lambda_{a}+\Delta \lambda n_{z}^{2}
\end{array}\right)_{i j k} \quad\left(\begin{array}{c}
\partial T / \partial x \\
\partial T / \partial y \\
\partial T / \partial z
\end{array}\right)_{i j k}
$$

where $\Delta \lambda=\lambda_{h}-\lambda_{a}$. In a cell fully occupied by the fluid $(\alpha=0)$ or by the solid $(\alpha=1)$, Eqs. (20) and (21) coincide with the conductivities of the respective substances, while in an interface cell, the apparent conductivity in the interfacial cell is given by the anisotropic matrix in Eq.(23). The above formula is only meaningful when constructing the discretised flux from the discretised temperature field, as it involves the spatial averaging of the local heat fluxes in the cell. The derivation and validations are detailed in Takeuchi et al. [2013] and Tsutsumi et al. [2014].

Note that the above Eulerian treatment of heat flux at the interface cell shows good compatibility with our immersed solid approach; the heat flux is simply obtained with the updated $\alpha$ and $\boldsymbol{n}$ as if the anisotropic heat conductivity distributes over the interfacial cells. 


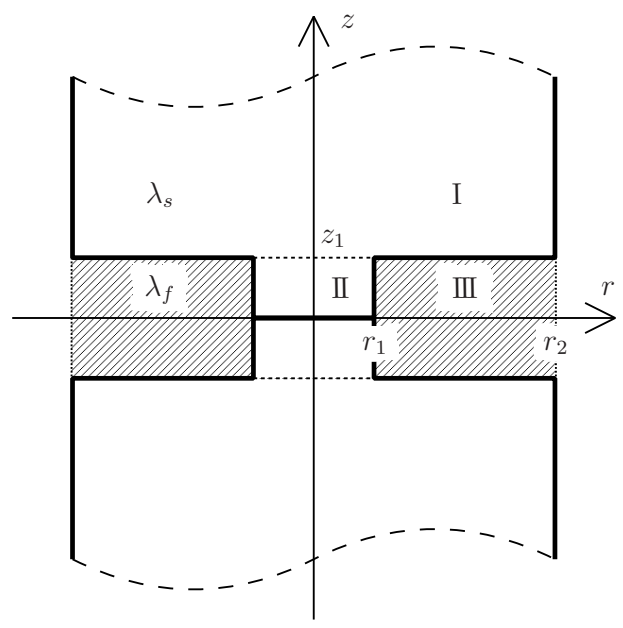

Figure 3: Schematic of a thermal resistance problem of a constricted solid object (plate in 2-D case and cylindrical shape in axisymmetric case). Two objects of width/diameter $2 r_{2}$ with a pair of protrusions of $2 r_{1}$ in width/diameter and $z_{1}$ in height contact with each other forming a constriction. Here, the shaded region (region III) is fluid. See also Fig. 17 for more specific configuration and symbols for 2-D case.

\subsection{Contact resistance model}

The thermal resistance model for contacting particles is summarised. In the present work, we follow the same approach as Sanokawa [1968a] to determine the thermal resistance at the contact site of the two particles in two- and three-dimensional problems. Sanokawa [1968a] modelled the contact site of two solid objects as a constricted cylinder immersed in a fluid environment, as schematically shown in Fig. 3. He solved the temperature distributions for the respective regions (I, II and III), and derived the exact form of the thermal resistance for the constricted cylindrical object. Here, by identifying the effective conductivities in the normal and tangential directions in the computational cell where the contact takes place, the contact heat flux is evaluated into a modified form of the interfacial heat flux $\boldsymbol{q}_{\mathrm{I}}$. We develop a 2-D model of thermal resistance of constricted plate and, for 3-D particulate flow, the axisymmetric model originally developed by Sanokawa [1968a] is employed with an addition of a missing term. The model assumes that the collision process takes place slowly enough that the elastic compression is a reversible process, and no thermo-elastic effect is included. The details of both 2-D and axisymmetric models are summarised in Appendix A, and, in the following, only the formulae used in the present simulation are summarised.

By solving the heat conduction equations in the zones I, II and III (Fig.3) together with the boundary conditions, the thermal resistance $R_{c}$ at the contact site $\left(-z_{1} \leq z \leq z_{1}\right)$ is commonly represented as

$$
R_{c}=\frac{2 r_{2}}{\lambda_{s}}\left(\frac{\mathscr{D}_{1}}{\mathscr{D}_{0}}+\frac{z_{1}}{r_{2}}\right)
$$




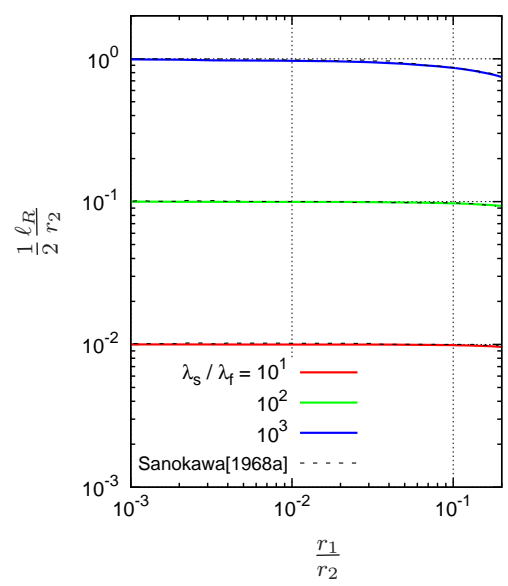

(a) $\frac{z_{1}}{r_{2}}=10^{-3}$

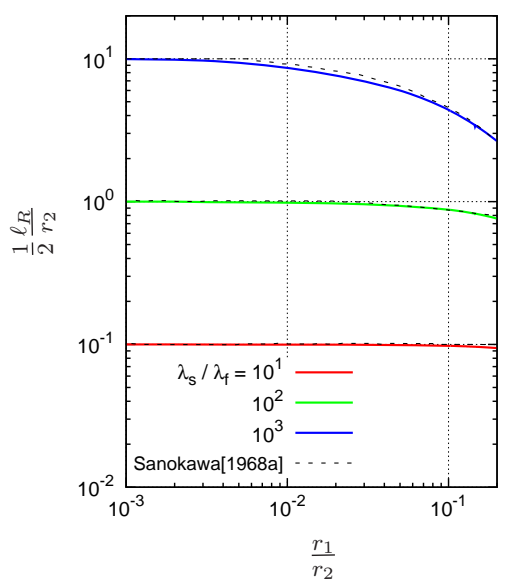

(b) $\frac{z_{1}}{r_{2}}=10^{-2}$

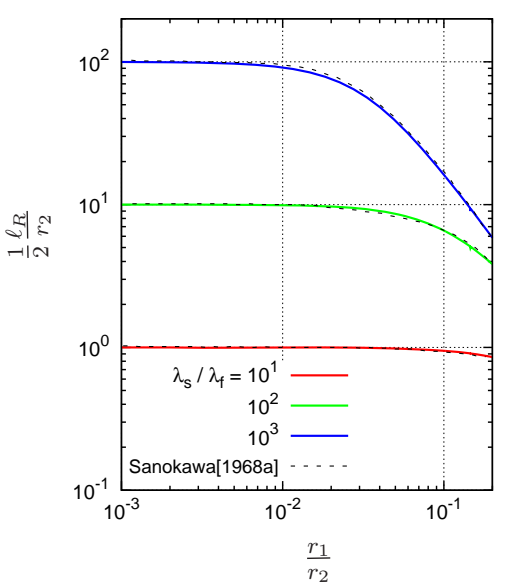

(c) $\frac{z_{1}}{r_{2}}=10^{-1}$

Figure 4: Dependence of the equivalent length of the thermal resistance at an axisymmetric constriction $\left(-z_{1} \leq z \leq z_{1}\right)$ on the constriction radius $\left(r_{1} / r_{2}\right)$ for different conditions of half-constriction heights $\left(z_{1} / r_{2}\right)$ and heat conductivities $\left(\lambda_{s} / \lambda_{f}\right)$.

regardless of the 2-D and axisymmetric situations. Here, $\mathscr{D}_{0}$ and $\mathscr{D}_{1}$ are the following determinants:

$$
\begin{aligned}
\mathscr{D}_{0}=\left|\begin{array}{c|cccccc}
a_{1,1} & \cdots & a_{1,2 j} & a_{1,2 j+1} & \cdots & a_{1,2 n} & a_{1,2 n+1} \\
\vdots & & \vdots & \vdots & & \vdots & \vdots \\
0 & \cdots & a_{2 i, 2 j} & 0 & \cdots & a_{2 i, 2 n} & a_{2 i, 2 n+1} \\
a_{2 i+1,1} & \cdots & a_{2 i+1,2 j} & a_{2 i+1,2 j+1} & \cdots & a_{2 i+1,2 n} & a_{2 i+1,2 n+1} \\
\vdots & & \vdots & \vdots & & \vdots & \vdots
\end{array}\right|(i, j=1, \cdots, n), \\
\mathscr{D}_{1}=\left|\begin{array}{ccccccc}
0 & \cdots & a_{1,2 j} & a_{1,2 j+1} & \cdots & a_{1,2 n} & a_{1,2 j+1} \\
\cdots & \vdots & \vdots & & \vdots & \vdots \\
0 & \cdots & a_{2 i, 2 j} & 0 & \cdots & a_{2 i, 2 n} & a_{2 i, 2 n+1} \\
b_{2 i+1} & \cdots & a_{2 i+1,2 j} & a_{2 i+1,2 j+1} & \cdots & a_{2 i+1,2 n} & a_{2 i+1,2 n+1} \\
\vdots & & \vdots & \vdots & & \vdots & \vdots
\end{array}\right|(i, j=1, \cdots, n),
\end{aligned}
$$

and the components of the determinants are given in Eqs.(34) and (39) of Appendix A for 2-D and axisymmetric cases, respectively. The size of the matrices of the determinants is dependent on the order of approximation $n$.

Thermal resistance is often represented with "equivalent length" calculated by the temperature drop due to the resistance divided by the temperature gradient at $z=z_{1}$ and $|r|<r_{1}$. The solid object of this thickness shows the equivalent effect in terms of the thermal resistance, which enables conversion of the thermal problem to geometric problem. In the present problems, the equivalent lengths $\ell_{R}$ for both 2-D and axisymmetric cases are formally given as:

$$
\frac{\ell_{R}}{r_{2}}=2\left(\frac{\mathscr{D}_{1}}{\mathscr{D}_{0}}+\frac{z_{1}}{r_{2}}\right)
$$




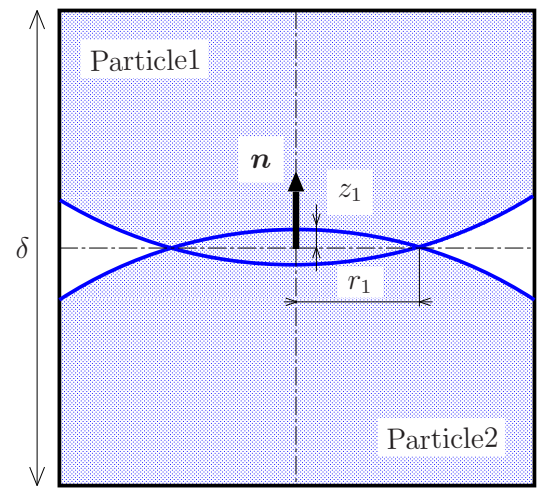

(a)

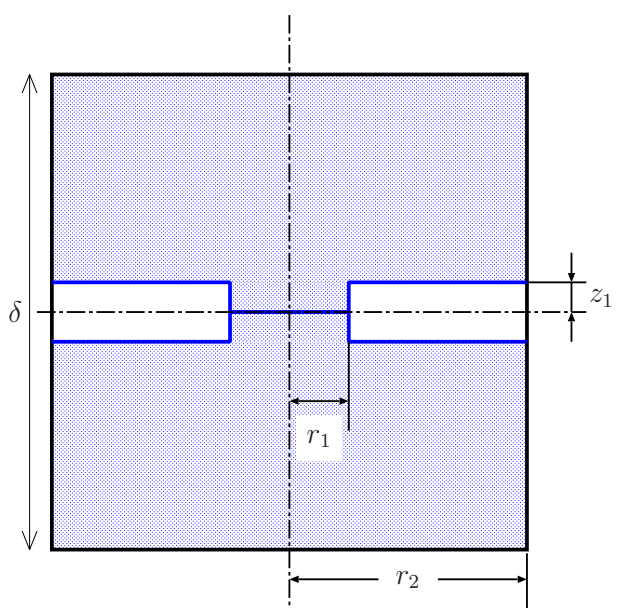

(b)

Figure 5: Schematic of the modelling of the heat transfer at the contact site of two particles. In the cell (cell size $\delta$ ) where a contact of two particles taking place (a), a heat transfer problem is solved as the heat through a constriction (b).

Taking $n=10$ for calculating Eqs. (25) and (26), Fig. 4 compares the equivalent lengths with the prediction by Sanokawa [1968a] (dashed lines) for axisymmetric cases for different values of the thermal and geometric parameters (i.e., $\lambda_{s} / \lambda_{f}, z_{1} / r_{2}$, respectively). Our results in the figure show reasonable agreement with those by Sanokawa [1968a], despite the truncations of the expansion series $(n=10)$. Considering the further comparisons by Sanokawa [1968a] to some experimental data under several combinations of metals and surrounding media, and also considering the typical values of $r_{1} / r_{2}\left(\sim 10^{-3}\right)$ and $z_{1} / r_{2}\left(\sim 10^{-2}\right)$ in the present simulations, the above result is acceptable. In the following, therefore, thermal equivalent length is calculated with $n=10$.

Note that for the 2-D model, in the limit of the point-contact $\left(r_{1} \rightarrow 0\right)$, the thermally equivalent length asymptotically reduces as $\ell_{R} \rightarrow 2 z_{1} \lambda_{s} / \lambda_{f}$, which is the same formula by Sanokawa [1968a] established for the axisymmetric case. This result yields that the heat flux at the constriction of infinitesimally small radius is $\lambda_{f} \Delta \Theta / 2 z_{1}$ (with $\Delta \Theta$ as the temperature gap across the constriction), which coincides with the observation by Batchelor and O'Brien [1977] that the heat flux of point-contact depends more sensitively on the conductivity of the fluid than that of the particles.

In the present study, the heat transfer in the cell including a contact site, Fig. 5(a), is modelled as a heat transfer problem through a constricted object as depicted in Fig. 5(b), assuming that the contacting diameter $2 r_{1}$ is small compared with the cell size $\delta$ and particle diameter $D_{p}$, and that the contact heat flux is strong in that direction (i.e., the centre-to-centre direction) and no heat substantially diffuses in the lateral direction for binary contacts. In the computational cell of the contact site, the interfacial heat flux, Eq.(23), is modified with the thermal resistance of the constriction. Here, we model that the solid object of the thermally equivalent length $\ell_{R}$ (and the width/diameter $2 r_{2}$ ) is virtually set in between the two particles 
in place of the constriction of height $2 z_{1}$. The effective conductivity of the cell in the normal direction is thereby given as:

$$
\lambda_{h}^{C}=\left(1-\frac{2 z_{1}}{\delta}+\frac{\ell_{R}}{\delta}\right)^{-1} \lambda_{s} .
$$

Here, $\delta$ is the grid spacing and we regard it as $2 r_{2}$. Considering that the Sanokawa model is derived for the solid objects aligned in the longitudinal direction and that the heat flux is only in the longitudinal direction, the tangential heat flux in the constricted heat transfer problem is assumed to be negligible in the present study. Therefore, we employ the following modified thermal conductivity in the tangential direction:

$$
\lambda_{a}^{C}=\left(1-\frac{2 z_{1}}{\delta}\right) \lambda_{s} .
$$

With Eqs.(28) and (29), the heat flux $\boldsymbol{q}_{\mathrm{I}}$ at the contact site is apparently represented with the same formula as Eqs. (22) and (23):

$$
-\boldsymbol{q}_{\mathrm{I}}^{C}=\left.\lambda_{h}^{C}(\boldsymbol{n n})_{i j k} \cdot \nabla T\right|_{i j k}+\left.\lambda_{a}^{C}(\boldsymbol{I}-\boldsymbol{n n})_{i j k} \cdot \nabla T\right|_{i j k}
$$

The contact radius and displaced distance are calculated by Hertzian contact theory [Timoshenko and Goodier 1970; Puttock and Thwaite 1969; Tripp 1985; Johnson 1987]. Along the line connecting the centres of the contacting two particles under a contacting load $f_{C_{n}}$, the Hertzian model is applied for determining the contact radius $r_{1}$ and height $z_{1}$, assuming that two elastic objects are statically in contact. This is justified as Timoshenko and Goodier [1970] that the duration of contact is very long in comparison with the period of lowest mode of vibration of the spheres, and the contact radius and height established for statical contact pressure are assumed to hold during the time which spheres remain in contact. For the particles in touch with a boundary wall, particle-wall collision is treated by taking the limit of the radius and elasticity of one particle to infinity, assuming that the solid wall deforms very little. As will be shown later, the order of magnitude of $r_{1} / \delta$ is found to range between $10^{-3}$ and $10^{-2}$, therefore, applying the Sanokawa model by regarding $2 r_{2}$ as $\delta$ would be accepted.

For estimating the contact radius, one possible approach is by a discrete element/particle method, which is often used for Lagrangian tracking of particles in a fluidized bed, and a collision treatment with the Hertzian contact model has been proposed [Tsuji et al. 1991]. However, it was reported that the non-linear spring model requires a small time increment for stability, which turns out an intensive computation. On the other hand, the collision model with a linear spring [Tsuji et al. 1993] guarantees invariant characteristic response time during the contact, and therefore, computationally less intensive. In the present study, by following the approach of Vargas and McCarthy [2002], a particle collision model with a linear spring [Tsuji et al. 1993] is employed for calculating the contacting force in the normal direction $f_{C_{n}}$, to focus on the establishment of a computational method for inter-particle heat transfer in a solid-dispersed two-phase flow, in a compatible form with the interfacial (fluid-particle) heat flux model [Takeuchi et al. 2013]. 


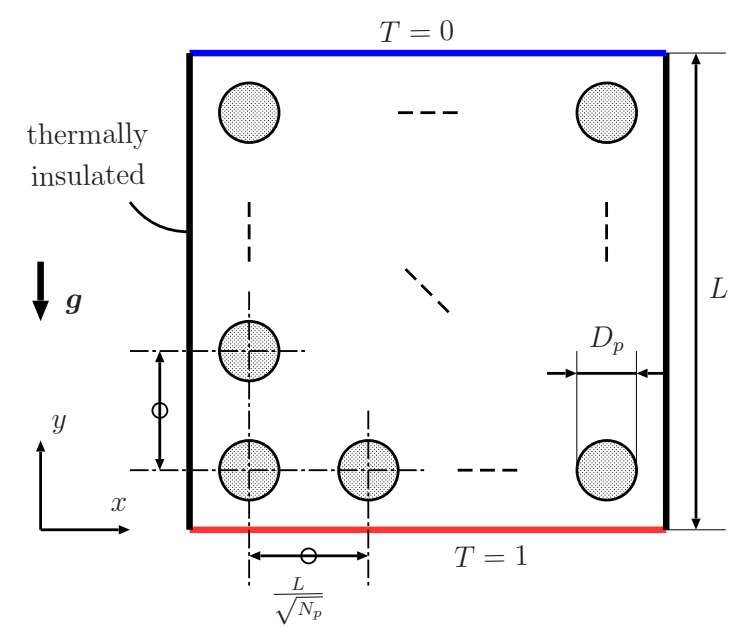

Figure 6: Schematic of the initial arrangement of particles in the computational domain and the boundary conditions.

\section{Effects of temperature distribution within finite-sized particles in 2-D particulate flow}

Prior to investigating the effect of thermal contact in solid-dispersed two-phase flows, a quick overview of the temperature distribution and solid behaviours under weak buoyancy is presented here for 2-D soliddispersed flows, based on the results of Takeuchi et al. [2013] and Tsutsumi et al. [2014]. In this section, the effect of the conduction within the individual particles is isolated, and the contact heat conduction in Section 2.4 is not accounted for.

A total of $14^{2}$ particles are initially arranged uniformly in a square domain (side length $L$ ) as show in Fig. 6. The corresponding bulk solid volume fraction is $38.5 \%$. The number of grid points is 200 in both $x$ and $y$ directions, and one particle diameter $D_{p}$ is resolved with ten grid points. The temperatures in the bottom and top walls are kept constant with a temperature difference $\Delta T=1$. Rayleigh number is set to $\mathrm{Ra}=10^{4}$ which is enough to cause natural convection in this system. To investigate the effect of natural convection on the particle behaviour, Prandtl number, density ratio and specific heat ratio are set to unity in this case. On the walls, the no-slip and Neumann conditions are applied for the fluid velocities and pressure, respectively, and a thermally-insulated boundary is assumed for the lateral walls. Here, by changing the ratio of conductivities $\left(\lambda_{s} / \lambda_{f}\right)$, we investigate the effect of the non-uniform temperature distribution inside the particles.

Figure 7(a) shows a typical snapshot of velocity and temperature fields together with the particles of under the conductivity ratio of $\lambda_{s} / \lambda_{f}=10^{-3}$ after reaching quasi-steady state. The fluid and particles are found to circulate in the clockwise direction; development of single circulation is typically observed in the cases of $\lambda_{s} / \lambda_{f}=10^{-3} \sim 10^{0}$ as well as the no-particle case (single-phase flow of the fluid). In the figure, the iso-thermal lines are drawn at the intervals of 0.1 . Note that the characteristic closed isotherms are 


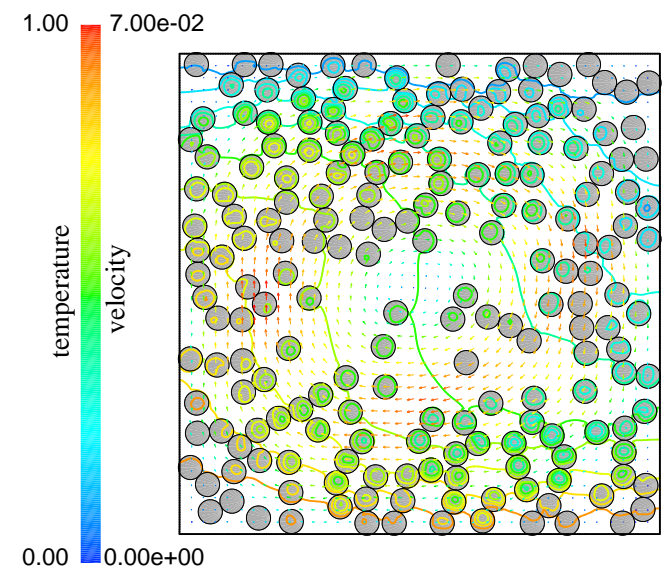

(a) $\lambda_{s} / \lambda_{f}=10^{-3}$

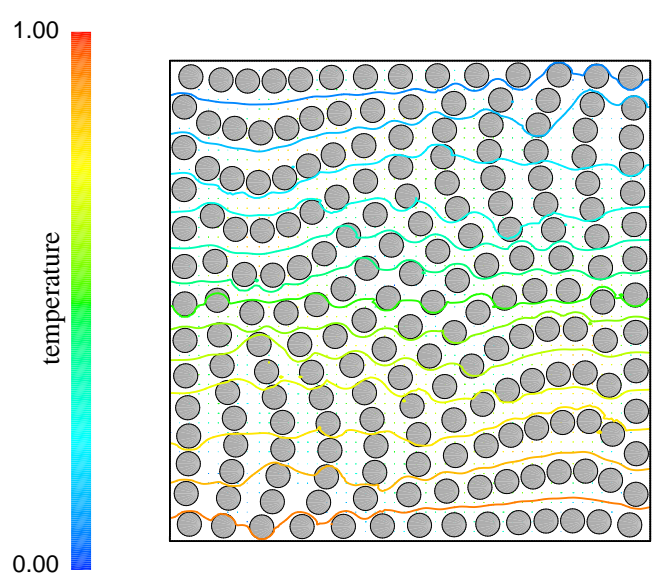

(b) $\lambda_{s} / \lambda_{f}=10^{3}$

Figure 7: Instantaneous velocity field and contours of temperature. Rayleigh number is set to be $10^{4}$, and the particles of conductivity ratios (a) $\lambda_{s} / \lambda_{f}=10^{-3}$ and (b) $\lambda_{s} / \lambda_{f}=10^{3}$ are employed.

observed inside the particles. This is due to the low conductivity of the particles; it takes a long time for those particles to reach steady temperature distribution in the environmental temperature field. However, the particles move before relaxing in the surrounding environment, causing the development of the temperature gradient within the particles. This characteristic temperature distribution is one of the typical effects of the thermal flow problems in solid-dispersed two-phase flow of finite-sized particles [Tsutsumi et al. 2014].

On the other hand, the highly-conductive particle $\left(\lambda_{s} / \lambda_{f}=10^{1} \sim 10^{3}\right)$, no matter where it moves, shows almost uniform temperature distribution within it. Another characteristic effect of the development of the temperature field inside and outside the particle is oscillating behaviour [Takeuchi et al. 2013] of the highly-conductive particles at the same Rayleigh number. A typical snapshot is shown in Fig. 7(b). This oscillating behaviour is explained that each particle experiences the hydrodynamic force driven by the buoyancy depending on the distance from the hot (bottom) wall, resulting in the restitution force in association with the conservation of angular momentum. The time scales for the thermal conduction in the dispersed phase (particle) and convection in the continuum phase (fluid) are responsible for the behaviour, and the balance between the time scales determines the oscillating frequency. The oscillatory behaviour of the fluid and particles is also the highlighted effect of the temperature distribution within the finite-sized particles in solid-dispersed two-phase flow.

In the following sections, let us look into characteristic effects of thermal contact including the heat conduction within the solid object. Also the behaviour of the particles and the heat transfer performance in the solid-dispersed two-phase flows are studied. 


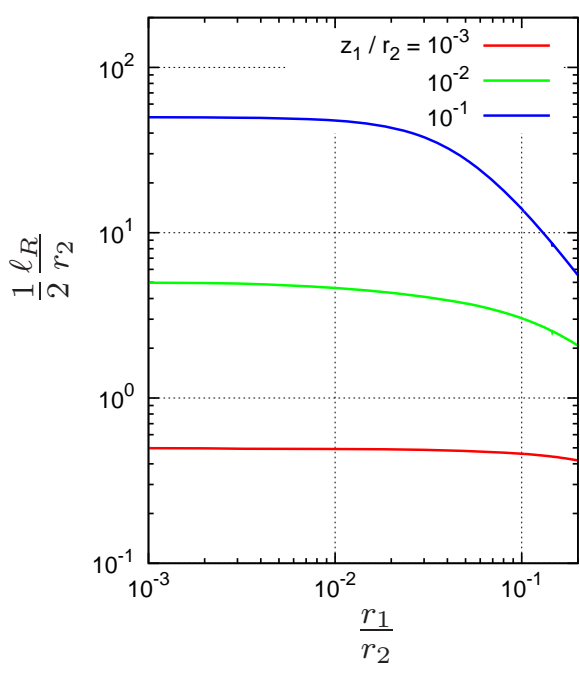

(a)

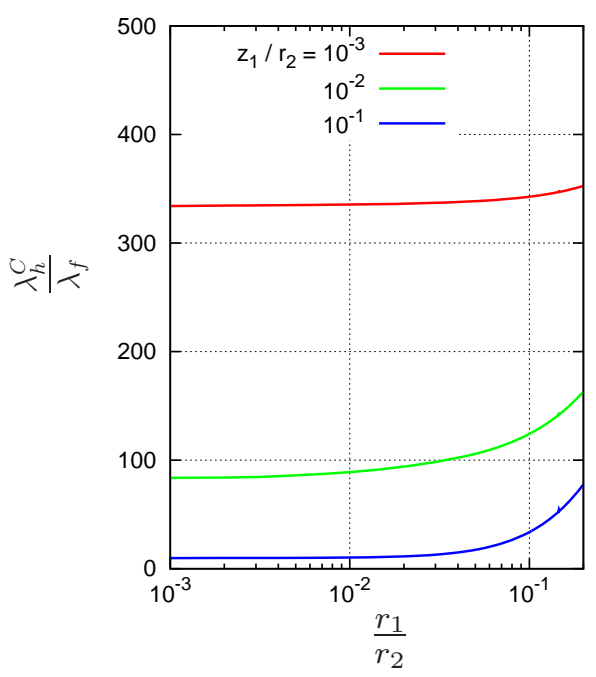

(b)

Figure 8: (a) Equivalent length of the thermal resistance and (b) effective heat conductivity in the normal direction with respect to the contact area for different $z_{1} / r_{2}$ values at a fixed conductivity ratio of $\lambda_{s} / \lambda_{f}=$ $5 \times 10^{2}$.

\section{2-D solid-dispersed flow with thermal contact effect}

The contact heat conduction model is demonstrated in 2-D solid-dispersed two-phase flow, since the interparticle heat flux is easily visualised. By setting a situation where frequent inter-particle (and particle-wall) collisions take place, the effect of the contacts is studied through looking at 2-D particulate flow structure and heat transfer in the system. As poorly-conducting particles are inert in terms of thermal contact, highlyconductive particles $\left(\lambda_{s} / \lambda_{f}=5 \times 10^{2}\right)$ are employed in the following. Also, to promote the occurrence of inter-particle contacts, a higher Rayleigh number is employed to induce stronger convection of the fluid.

\subsection{Contacting area and the contact heat transfer for stationary particles}

Basic characteristics of contacting area and contact heat transfer are investigated for stationary particles.

Figure 8(a) compares the equivalent lengths of the thermal resistance as a function of constriction width for different $z_{1} / r_{2}$ values, and Fig. 8(b) shows the dependence of the effective heat conductivity in the normal direction based on the equivalent lengths in Fig. 8(a). For the smallest value of the normalised halfconstriction height, $z_{1} / r_{2}=10^{-3}$, the effective conductivity in the normal direction is rather insensitive to $r_{1} / r_{2}$, while, for the cases of $z_{1} / r_{2}=10^{-2}$ and $10^{-1}$, significant increases in the effective conductivity are observed in the radial range of $r_{1} / r_{2}>10^{-2}$. The result in Fig. 8 suggests a non-negligible effect of the direct contact conduction for a contacting radius $r_{1}$ larger than $10^{-2} \delta$. 


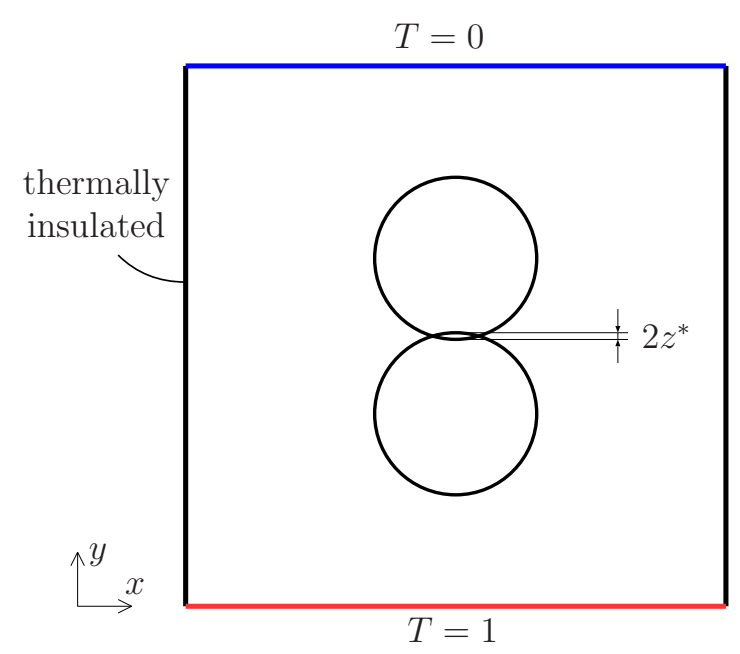

Figure 9: Schematic of the arrangement of the contacting particles in the computational domain.

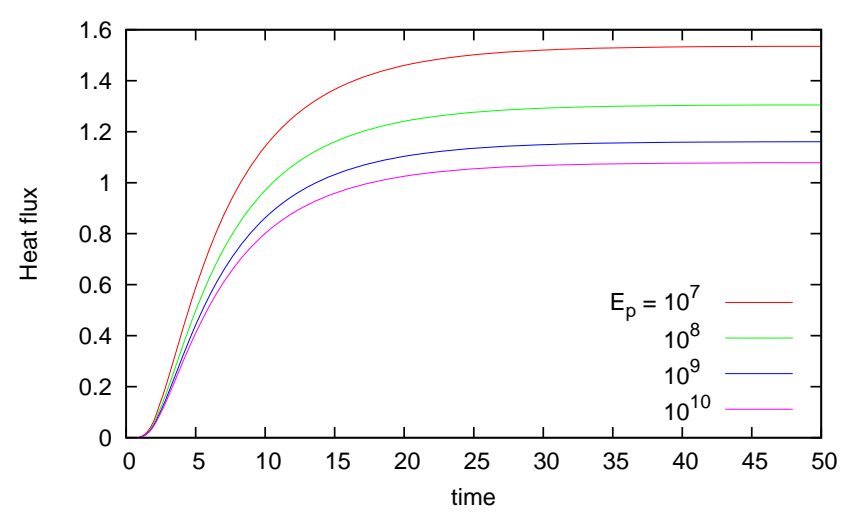

Figure 10: Time evolutions of the heat flux through the contact surface in a system of two contacting particles in two dimensions. The fixed overlapping distance $2 z^{*}$ (see Fig.9) is $0.01 \delta$.

The thermal contact model is applied to a system of two particles in static contact. As shown in Fig. 9, the particles are fixed with an overlapping distance $2 z^{*}$. The initial temperature in the domain is $T_{0}$ everywhere, and the convection of the surrounding fluid is turned off in this problem. The number of grid points is 50 in both $x$ and $y$ directions, and ten grid points are given to cover one particle diameter. The overlapping distance $2 z^{*}$ is set to be $0.01 \delta$. The ratio of heat conductivities is $\lambda_{s} / \lambda_{f}=5 \times 10^{2}$. The normalised Young's modulus $E_{p}$ of the particles is varied in the range from $10^{7}$ to $10^{10}$, and, with a fixed Poisson ration 0.3 , the corresponding values of $r_{1} / r_{2}$ are found to be $1.02 \times 10^{-1}, 4.70 \times 10^{-2}, 2.20 \times 10^{-2}$ and $1.00 \times 10^{-2}$, respectively.

Figure 10 shows the simulation results of the heat flux in $y$ direction through the contacting area. The figure shows that the transferred heat becomes larger with decreasing the Young's modulus, and in turn, with increasing the contacting area. 


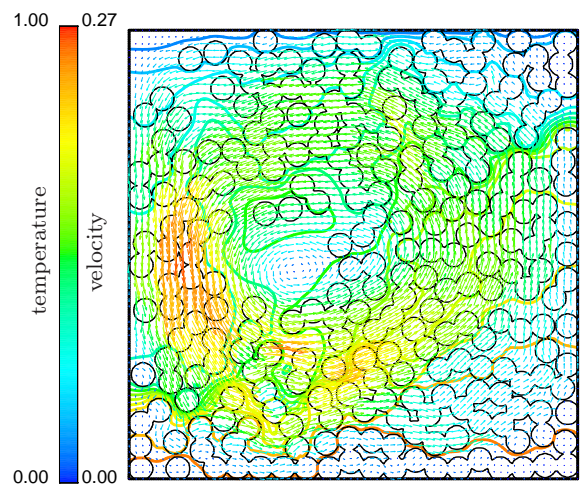

(a) Velocity field

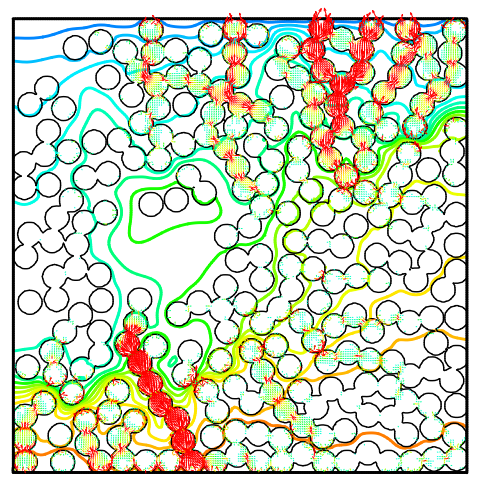

(b) Conductive heat flux

Figure 11: Instantaneous flow field and heat flux at Rayleigh number $10^{6}$ for the Young's modulus $E_{p}=$ $10^{7}$ after reaching quasi-steady state (at $t=1000$ ). Bulk solid volume fraction is $50.3 \%$. (a) Velocity and temperature fields. (b) Heat flux vectors (arrows) at the same instance as (a). The density ratio is $\rho_{s} / \rho_{f}=1.005$. The isothermal lines are drawn at constant intervals.

\subsection{Heat transfer in solid-dispersed flow with contact heat transfer}

Under a strong natural convection where inter-particle collision takes place frequently, we look into a gross heat transfer performance of the system involving contact heat transfer. For setting up a situation of sufficient collision frequency of particles in a relatively strong thermally-driven flow, Rayleigh number Ra and the number of particles are set to $\mathrm{Ra}=10^{6}$ and $N_{p}=16^{2}$, respectively. The corresponding bulk solid volume fraction is $50.3 \%$. The heat conductivity of the particles are fixed to be $\lambda_{s} / \lambda_{f}=5 \times 10^{2}$ in the present case. The Young's modulus and Poisson ratio are $E_{p}=10^{7}$ and 0.3 , respectively. The same domain size and initial particle arrangement as Fig. 6 are employed, and the boundary conditions are also the same.

A single circulating flow is found to develop around the domain centre (including the cases of $E_{p}$ values between $10^{7}$ and $10^{10}$ ), and no oscillation mode (as for case of $\mathrm{Ra}=10^{4}$ in the previous section) is observed. Figure 11(a) shows instantaneous velocity and temperature fields for the case of $E_{p}=10^{7}$ at $t=1000$. A counter-clockwise circulating flow is observed. There is a region of low number density of particles slightly off-centre of the domain, and resulting in rather concentrated regions near the walls and corners. However, in the present case, the convection for the higher Rayleigh number causes locally stronger circulating velocity field in a densely populated region of the highly-conductive particles.

The increased effect of the contact frequency appears strongly in the inter-particle heat flux. Figure 11(b) shows the heat flux field (visualised with arrows) at the same instance as Fig. 11(a). In the figure, strong heat fluxes are observed along the chained structures of the particles, particularly in the bottom-left and top-right regions of the domain. This is because the counter-clockwise circulation generates the densely-populated regions, giving rise chained structures of the particles stemming from/to the bottom/top (hot/cold) wall. 


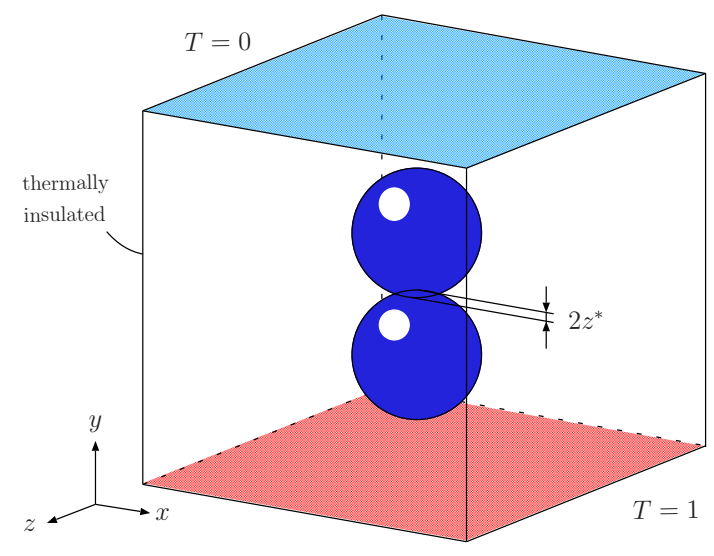

Figure 12: Schematic of arrangement of spherical particles in contact.

This chained structure is an efficient heat-transfer path through the particles, and branching of the path sometimes causes unequal distributions of heat flux depending on the temperatures of the particles and surrounding fluid. It is also remarkable that the burst fluxes through the chained-particles cross a number of isothermal lines, causing the regions of large temperature gradient in the bottom-left and top-right corners. In the preliminary study under $\mathrm{Ra}=10^{5}$ and $N_{p}=14^{2}$, only weaker burst flux is observed.

By the present thermal contact model, sequential heat transfer across the several diameters of the particles is observed, which also illustrates the effects of the temperature gradients within the individual particles and over the multiple particles. This characteristic effect of inter-particle thermal contact may work to enhance the mixing in the solid-dispersed two phase flow in three dimensions through the lateral contacts of particles.

\section{3-D solid-dispersed two-phase flow with contact heat transfer}

The interfacial flux model and thermal contact conduction model are applied to three-dimensional problem. In the following three dimensional natural convection with particles, by separating the heat flux into the contributions by convection and conduction, the change in heat transfer mode is studied for different bulk solid volume fractions under a condition of a fixed heat conductivity ratio.

\subsection{Basic characteristics of contacting area and the contact heat transfer}

To see the thermal response in the particulate system in three dimensions, two spherical particles are fixed with an overlapping distance of $2 z^{*}=0.01 \delta$ in a cubic domain, as Fig. 12 shows. A constant temperature difference is maintained between the top and bottom walls, and an insulated condition is applied for the four lateral walls. The diameter of the particles is one fifth of the domain side length, and ten grid points are allocated for one particle diameter. The conduction problem is solved without the fluid convection. 


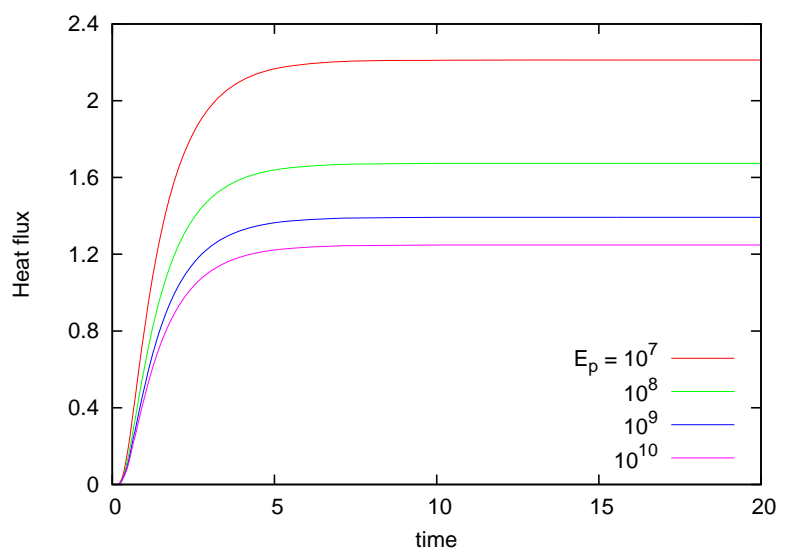

Figure 13: Time evolutions of the heat flux through the contact surface in a system of two contacting particles in three dimensions. The fixed overlapping distance $2 z^{*}$ (see Fig.12) is $0.01 \delta$.

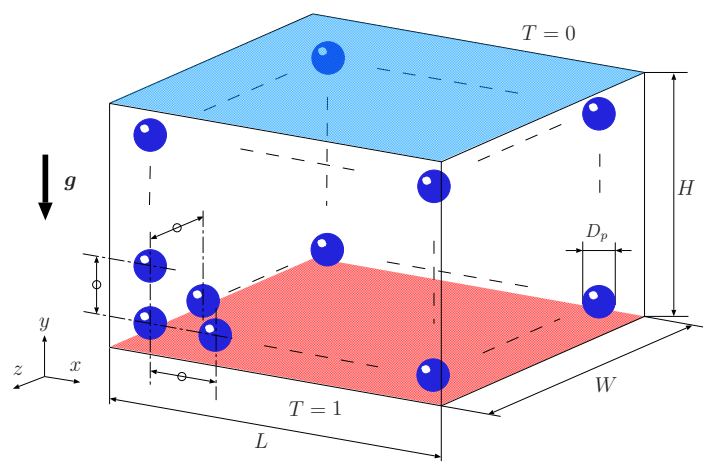

Figure 14: Schematic of the initial arrangement of particles in the computational domain.

Young's modulus is varied in the range between $10^{7}$ and $10^{10}$ at the intervals of $10^{1}$, and the corresponding contacting radii are found to be $r_{1} / r_{2}=1.65 \times 10^{-1}, 7.65 \times 10^{-2}, 3.55 \times 10^{-2}, 1.65 \times 10^{-2}$, respectively.

The time variations of the heat flux through the contact area are plotted in Fig. 13. As observed in 2-D cases, lower elasticity also causes larger contacting radius in 3-D, and in turn, the heat flux through the contact site. However, the heat fluxes in the figure are more pronounced than in the 2-D case due to the dimensional effect.

\subsection{Effects of the solid volume fraction and thermal conductivity of the particles}

Heat transfer in solid-dispersed flow is solved in a fully three-dimensional system, and the change of the major modes of heat transfer is studied for different particle numbers and conductivities.

As schematically shown in Fig. 14, the particles are initially arranged at the regular intervals in all the directions. The number of particles $N_{p}$ are varied exponentially as $N_{p}=9,243,1125$ and 3087. The corresponding bulk solid volume fractions are $0.102 \%, 2.80 \%, 12.8 \%$ and $35.1 \%$, respectively. The computational cells are uniformly distributed, and the number of grid points are 256,80 and 256 in $x, y$ and $z$ 

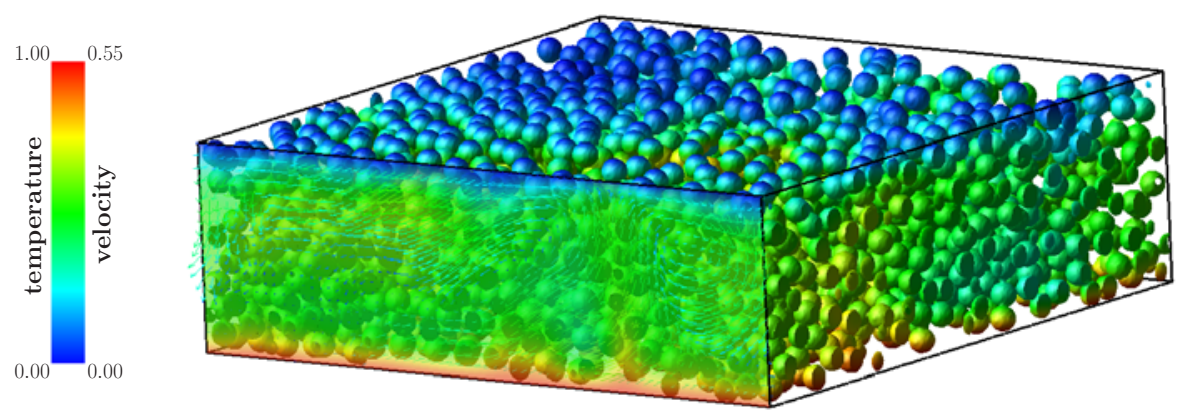

(a) $\lambda_{s} / \lambda_{f}=10^{0}$
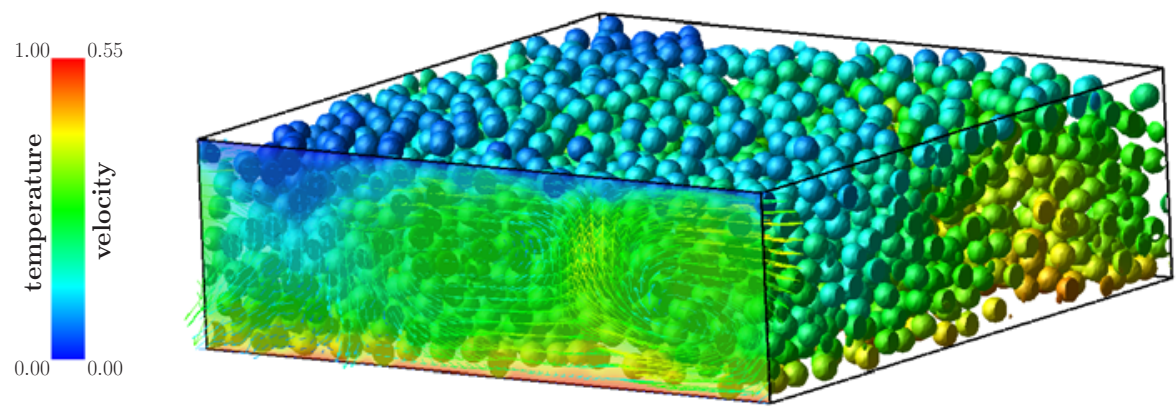

(b) $\lambda_{s} / \lambda_{f}=10^{2}$

Figure 15: Instantaneous flow fields $(t=800)$ visualised by velocity vectors (in a vertical face of the domain) and local surface temperature of particles of conductivity ratios (a) $\lambda_{s} / \lambda_{f}=10^{0}$ and (b) $\lambda_{s} / \lambda_{f}=$ $10^{2}$. A total of 3087 particles are employed for each case.

directions, respectively. One particle diameter is resolved by ten grid points. With a constant temperature difference between the top and bottom walls, the Rayleigh number is set to be $10^{5}$. Time-average is taken from $t=200$ (after reaching quasi-steady state) to 800 in non-dimensional time.

Figure 15 compares two snapshots of the solid-dispersed flow for different conductivity ratios of $\lambda_{s} / \lambda_{f}=$ $10^{0}$ and $10^{2}$. A total of 3087 particles are employed for each case. The velocity and temperature fields in a vertical coss-section are visualised in the figure, and the local surface temperature of the particles is also illustrated. For all the conditions, the thermal convection causes strong mixing of the fluid and particles, and characteristic temperature distributions are observed: the temperature distribution of the highly-conductive $\left(\lambda_{s} / \lambda_{f}=10^{2}\right)$ particle is almost uniform over each particle surface, whereas for the neutrally-conductive $\left(\lambda_{s} / \lambda_{f}=10^{0}\right)$ case, the particles show non-uniform distributions of surface temperature, particularly for those near the top and bottom walls.

To investigate the major heat transfer mode, the local heat flux in the domain is decomposed into the two contributions: convection and conduction. Note that heat transfer by moving individual particle is included into the contribution by the convective component. Figure 16 compares the convective and conductive heat fluxes averaged over the horizontal cross-sections and time for different conditions of solid volume fractions 


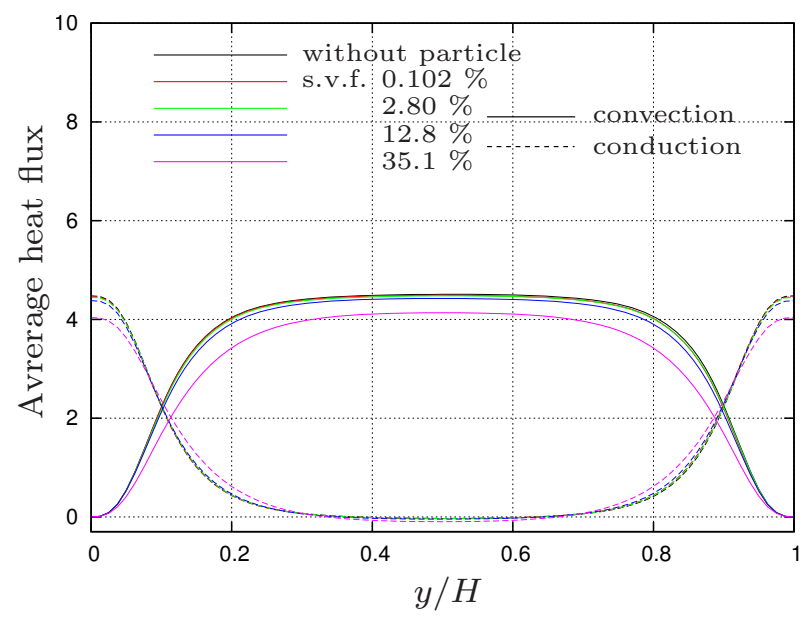

(a) $\lambda_{s} / \lambda_{f}=10^{0}$

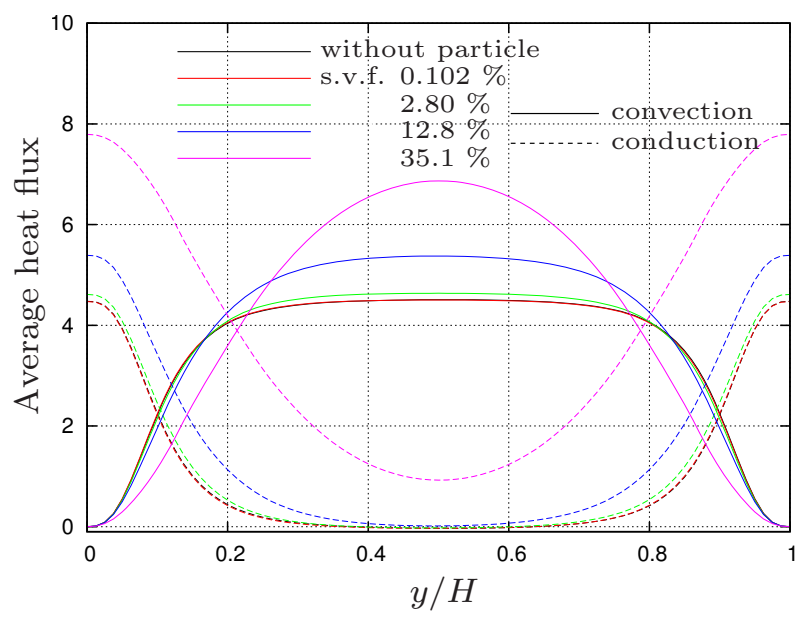

(b) $\lambda_{s} / \lambda_{f}=10^{2}$

Figure 16: Vertical distribution of averaged heat flux decomposed into the contributions by conduction and convection. Plotted for different solid volume fractions (s.v.f.) and $\lambda_{s} / \lambda_{f}$ values.

and $\lambda_{s} / \lambda_{f}$ values. In both $\lambda_{s} / \lambda_{f}$ cases, the convective component (represented by solid lines) increases in the middle of the domain, while in the near-wall regions the conduction (dashed lines) is dominant. The trends of the near-wall conductive role of the highly-conductive particles (and the convective components for both $\lambda_{s} / \lambda_{f}$ cases) are comprehensible. The above conductive contribution for the neutrally- (and poorly) conductive particles is explained by following the view obtained in 2-D simulation [Tsutsumi et al. 2014]: because the energy input from the hot wall is mainly consumed to raise the particle temperature in the nearwall region and the less amount of energy is transferred to convection in the middle of the domain, which produces inactive particles, especially near the walls, and those particles further inhibit the energy transfer from/to the fluid phase to/from the wall.

Also from Fig. 16, in the case of $\lambda_{s} / \lambda_{f}=10^{0}$, the convective component decreases monotonically as the solid volume fraction increases, and the fraction of the contribution by conduction becomes relatively large. On the other hand, in the case of $\lambda_{s} / \lambda_{f}=10^{2}$, the convective contribution increases as the solid volume fraction increases, and at the same time, the conductive contribution increases, in the range of the solid volume fraction studied here. This is explained that the convection is strong enough with the neutrallyconductive $\left(\lambda_{s} / \lambda_{f}=10^{0}\right)$ particles and the convection determines the basic level of the transported heat, while, for the case of the highly-conductive particles $\left(\lambda_{s} / \lambda_{f}=10^{2}\right)$, the contribution by the conductive component is superposed on top of the convective heat transfer.

In the heat transfer problem in 3-D solid-dispersed flows, some similarities to 2-D case are observed by further varying bulk solid volume fraction and the heat conductivity of the particles. Our independent 2-D study shows that, by adding the highly-conductive particles until the free convection of the particles 
is almost disabled, the conductive contribution becomes predominant, resulting in the decrease in average Nusselt number. Our preliminary 3-D simulations show the similar transition of the heat transfer mode by further adding the particles. Also, Fig. 16(a) shows a region of negative contribution of conduction in the range of $0.3 \leq y / H \leq 0.7$ for the dense case with $\lambda_{s} / \lambda_{f}=10^{0}$ particles. The result suggests a region of negative temperature gradient, and the Lagrangian heat transport by the individual particles is responsible for this. Heat transfer problem in 2-D and 3-D packed beds in association with adverse temperature gradient is the ongoing subject of research by the present authors.

\section{Conclusion}

A contact conductance model was developed for heat transfer problem in a solid-dispersed two-phase flow including finite-sized particles, based on analytical solutions for 2-D and axisymmetric constricted objects. The models are described in a geometric form, which enables incorporation into the heat flux model at the fluid-solid interface and construction of a general formula of interfacial heat flux model. The interaction between the fluid and particle was treated by our original immersed solid method of explicit predictorcorrector solver.

The numerical method was applied to natural convection with finite-sized particles in closed domain. The contact increases the local heat flux around the contacting site, and the overall heat transfer performance is increased. Most remarkable effect was observed that, when a chained structure of particles is formed in a circulating particulate flow, a burst heat flux takes place across several particle diameters. This is one of the characteristic effects of the heat transfer in two-phase flow with finite-sized particles, i.e., heat fluxes within the particles as well as across the contacting particles.

The contributions of the convective and conductive components of the heat flux were studied on the gross heat transfer in the particulate flow for different solid volume fractions and heat conductivities of the particles. For neutrally-conductive particles, the balance between the conductive and conductive components remains approximately the same irrespective of the solid volume fraction, suggesting that the basic level of the transferred heat is determined by the convection. On the other hand, with highly-conductive particles, the conductive component was found to enhance the overall amount of heat transfer in the particulate flows as the solid volume fraction is increased. Also, the adverse contribution of conduction for neutrally-conductive case and the suppress of the conductive contribution by further increase of the highlyconductive particles suggest interesting implications for the change of the major heat transfer paths in the particulate flows depending on the material property and mobility of the particles. 


\section{References}

Batchelor and O'Brien (1977) "Thermal or electrical conduction through a granular material", Proceedings of the Royal Society of London. Series A, Mathematical and Physical Sciences 355, No.1682, pp.313333

Fenech, H. and Rohsenow, W.M. (1963) "Prediction of thermal conductance of metallic surfaces in contact", Trans. ASME, Journal of Heat Transfer 85(1) pp.15-24

Feng, J., Hu, H.H. and Joseph, D.D. (1994) "Direct simulation of initial value problems for the motion of solid bodies in a Newtonian Fluid. Part 2. Couette and Poiseuille flows", Journal of Fluid Mechanics 277 pp.271-301

Feng, Z.-G. and Michaelides, E.E. (2000) "A numerical study on the transient heat transfer from a sphere at high Reynolds and Peclet numbers", International Journal of Heat and Mass Transfer 43, pp.219-229

Holm, R., (1967) Electric contacts : theory and application (4th completely rewritten ed.), Springer-Verlag, Berlin

Johnson, K.L. (1987) Contact mechanics, Cambridge University Press

Kajishima, T., Takiguchi, S., Hamasaki, H. and Miyake, Y. (2001) "Turbulence structure of particle-laden flow in a vertical plane channel due to vortex shedding", JSME International Journal Series B 44-4, pp.526-535.

Kajishima, T. and Takiguchi, S. (2002) "Interaction between particle clusters and particle-induced turbulence", International Journal of Heat and Fluid Flow, 23-5, pp.639646

Kajishima, T. (2004) "Influence of particle rotation on the interaction between particle clusters and particleinduced turbulence", International Journal of Heat and Fluid Flow 25-5, pp.721-728

Kim, J. and Choi, H. (2004) "An immersed-boundary finite-volume method for simulation of heat transfer in complex geometries", KSME International Journal 18, pp.1026-1035

Kim, J., Kim, D. and Choi, H. (2001) "An immersed-boundary finite-volume method for simulation of flow in complex geometries", Journal of Computational Physics 171, pp.132-150

Madhusudana, C.V. (1996) Thermal Contact Conductance, Springer 
McKenna, T.F., Dupuy, J. and Spitz, R. (1995) "Modeling of transfer phenomena on heterogeneous Ziegler catalysts: Differences between theory and experiment in olefin polymerization (an introduction)", Journal of Applied Polymer Science 57, Issue 3, pp.371-384

McKenna, T.F., Spitz, R. and Cokljat, D. (1999) "Heat transfer from catalysts with computational fluid dynamics", Reactors, Kinetics, and Catalysis 45-11, pp.2392-2410

Moukalled, F., and Darwish, M. (1997) "New bounded skew central difference scheme, Part II: Application to natural convection in an eccentric annulus", Numerical Heat Transfer Part B 31, pp.111-133

Nishiura, D., Shimosaka, A., Shirakawa, Y. and Hidaka, J. (2006) "Hybrid simulation of hindered settling behavior of particles using discrete element method and direct numerical simulation” (in Japanese), Kagaku Kogaku Ronbunshu, 32-4, pp.331-340

Pacheco, J.R., Pacheco-Vega, A., Rodić, T. and Peck, R.E. (2005) "Numerical simulations of heat transfer and fluid flow problems using an immersed-boundary finite-volume method on nonstaggered grids", Numerical Heat transfer, Part B 48, pp.1-24

Pacheco-Vega, A., Pacheco, J. R. and Rodić, T. (2007) "A general scheme for the boundary conditions in convective and diffusive heat transfer with immersed boundary methods", Trans. ASME, Journal of Heat Transfer 129-11, pp.1506-1516

Puttock, M.J. and Thwaite, E.G. (1969) "Elastic Compression of Spheres and Cylinders at Point and Line Contact", National Standards Laboratory Technical Paper No.25, Commonwealth Scientific and Industrial Research Organization, Australia

Ren, W.W., Shu, C., Wu, J. and Yang, W.M. (2012) "Boundary condition-enforced immersed boundary method for thermal flow problems with Dirichlet temperature condition and its applications", Computers \& Fluids 57, pp.40-51

Sakurai, T., Minami, T., Kawaguchi, T., Tanaka, T., Tsuji, T. and Tsuji, Y. (2007) 'DEM Simulation and inftrared thermography measurement of fluidezed bed with heat transfer", Proceedings of 6th International Conference on Multiphase Flow (ICMF2007), CD-ROM Paper No. S2_Fri_A_62

Sakurai, T., Minami, T., Kawaguchi, T., Tsuji, T., Tanaka, T. and Tsuji, Y. (2009) ”DEM-CFD simulation and thermography measurement of fluidized bed with heat transfer" (in Japanese), Transaction of JSME Ser.B 75-753, pp.1041-1048

http://ci.nii.ac.jp/naid/110007226534/en/ 
Sanokawa, K. (1968) "Heat transfer between metallic surfaces in contact : 1st report, the conditions of both metals in contact being the same", Bulletin of JSME 11-44, pp.253-263

http://dx.doi.org/10.1299/jsme1958.11.253

Sanokawa, K. (1968) "Heat transfer between metallic surfaces in contact : 2nd report, the effects of the thickness of metal, and the conditions of both metals in contact being different” , Bulletin of JSME 11 No.44, pp.264-275

http://dx.doi.org/10.1299/jsme1958.11.264

Sanokawa, K. (1968) "Heat transfer between metallic surfaces in contact : 3rd report, the effect of oxidation of contacting surfaces", Bulletin of JSME 11 No. 44, pp.276-286

http://dx.doi.org/10.1299/jsme1958.11.276

Sanokawa, K. (1968) "Heat transfer between metallic surfaces in contact: 4th report, the effects of the shape of surface roughness and the waviness, and the approximate method of calculating thermal contact resistance”, Bulletin of JSME 11 No.44, pp.287-293

http://dx.doi.org/10.1299/jsme1958.11.287

Sun, J. and Chen, M.M. (1988) "A theoretical analysis of heat transfer due to particle impact", Int. J. Heat and Mass Transfer 31-5, pp.969-975

Takeuchi, S., Tsutsumi, T. and Kajishima, T. (2013) 'Effect of temperature gradient within a solid particle on the rotation and oscillation modes in solid-dispersed two-phase flows", International Journal of Heat and Fluid Flow 43, pp.1525

Timoshenko, S.P. and Goodier, J.N. (1970) Theory of elasticity (3rd ed.), McGraw-Hill

Tripp, J.H. (1985) ”Hertzian Contact in Two and Three Dimensions”, NASA Technical Paper 2473

Tsuji, Y., Tanaka,T. and Ishida, T. (1992) 'Lagrangian numerical simulation of plug flow of cohesionless particles in a horizontal pipe", Powder Technology 71, pp.239-250

Tsuji, Y., Kawaguchi, T. and Tanaka, T. (1993) "Discrete particle simulation of two-dimensional fluidized bed", Powder Technology 77, pp.79-87

Tsutsumi, T., Takeuchi, S. and Kajishima, T. (2014) "Heat transfer and particle behaviours in dispersed twophase flow with different heat conductivities for liquid and solid", Flow Turbulence and Combustion 92-1-2, pp.103-119 
Ueyama, A., Moriya, S., Nakamura, M. and Kajishima, T. (2011) "Immersed boundary method for liquidsolid two-phase flow with heat transfer" (in Japanese), Transaction of JSME Series B, 77-775, pp.803814

Vargas, W.L. and McCarthy, J.J. (2001) "Heat conduction in granular materials", AIChE Journal 47, No.5, pp.1052-1059

Vargas, W.L. and McCarthy, J.J. (2002) "Conductivity of granular media with stagnant interstitial fluids via thermal particle dynamics simulation”, International Journal of Heat and Mass Transfer 45, pp.48474856

Yovanovich, M.M. (1967) "Thermal contact resistance across elastically deformed spheres", J. Spacecraft 4 No.1, pp.119-122

Yu, Z., Shao, X. and Wachs, A. (2006) "A fictitious domain method for particulate flows with heat transfer", Journal of Computational Physics 217, pp.424-452

Yuki, Y., Takeuchi, S. and Kajishima, T. (2007) "Efficient immersed boundary method for strong interaction problem of arbitrary shape object with the self-induced flow", Journal of Fluid Science and Technology 2-1, pp.1-11 


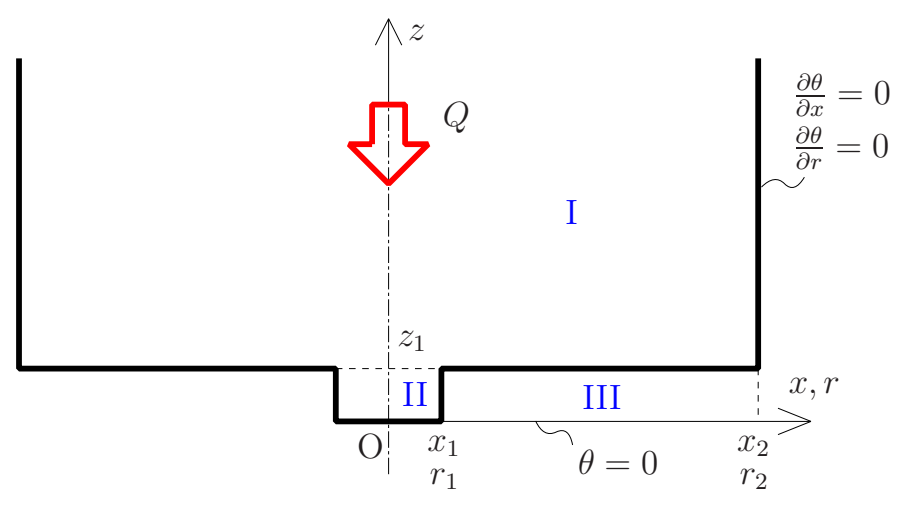

Figure 17: Schematic of constricted solid objects (top half), and the boundary conditions for solving the thermal resistance problem through the constriction.

\section{Appendix A Thermal resistance models}

Thermal heat resistance models for a constricted solid object are presented for two-dimensional and axisymmetric situations. The two-dimensional case is newly developed, and the axisymmetric case is derived by following the formulation of Sanokawa [1968a] and presented with a neglected term.

A pair of half-infinite plate/cylinder with a protrusion are arranged as Fig.17, and the thermal resistances at the constriction are analysed under a constant heat flux $Q$, assuming that, in the situation of contacting particle, a constant normal heat flux is maintained during the course of contact; since the interior temperature could distribute in a complex manner as Fig. 7(a) shows, the iso-flux condition is more realistic in the present study rather than a condition of constant temperature difference. The domain is divided into the three regions, I, II and III. The regions I and II are solid of the thermal conductivity $\lambda_{s}$, and region III is occupied by the fluid of thermal conductivity $\lambda_{f}$. In the following, the temperature is denoted by $\theta_{i}(i=\mathrm{I}, \mathrm{II}, \mathrm{II})$.

\section{A.1 Two-dimensional case}

The heat conduction equations, $\nabla^{2} \theta_{i}=0(i=\mathrm{I}, \mathrm{II}, \mathrm{II})$, are solved under the following boundary conditions:

$$
\begin{array}{lll}
z \geq z_{1} \quad: \quad & Q=\int_{-x_{2}}^{x_{2}} \lambda_{s} \frac{\partial \theta_{\mathrm{I}}}{\partial z} \mathrm{~d} x, \\
z=0 \quad: & \theta_{\mathrm{II}}=0 \text { and } \theta_{\mathrm{III}}=0, \\
z=z_{1} \quad: \quad & \theta_{\mathrm{I}}=\theta_{\mathrm{II}} \text { and } \lambda_{s} \frac{\partial \theta_{\mathrm{I}}}{\partial z}=\lambda_{s} \frac{\partial \theta_{\mathrm{II}}}{\partial z}, \quad\left(0 \leq x \leq x_{1}\right) \\
& \theta_{\mathrm{I}}=\theta_{\mathrm{III}} \text { and } \lambda_{s} \frac{\partial \theta_{\mathrm{I}}}{\partial z}=\lambda_{f} \frac{\partial \theta_{\mathrm{II}}}{\partial z}, \quad\left(x_{1} \leq x \leq x_{2}\right) \\
x=x_{1} \quad: \quad \theta_{\mathrm{II}}=\theta_{\mathrm{III}} \text { and } \lambda_{s} \frac{\partial \theta_{\mathrm{II}}}{\partial x}=\lambda_{f} \frac{\partial \theta_{\mathrm{II}}}{\partial x}, & \\
x=x_{2} \quad: \quad & \frac{\partial \theta_{\mathrm{I}}}{\partial x}=0 \text { and } \frac{\partial \theta_{\mathrm{II}}}{\partial x}=0 .
\end{array}
$$


The general solutions for the respective regions are as follows:

$$
\begin{aligned}
\theta_{\mathrm{I}} & =\Theta_{0}+\frac{Q}{2 \lambda_{s} x_{2}} z+\sum_{j=1} A_{j} \exp \left(-j \pi \frac{z-z_{1}}{x_{2}}\right) \cos \left(j \pi \frac{x}{x_{2}}\right) \\
\theta_{\mathrm{II}} & =\left(\frac{\Theta_{0}}{z_{1}}+\frac{Q}{2 \lambda_{s} x_{2}}\right) z+\sum_{j=1} B_{j} \sinh \left(\alpha_{j} \frac{z}{x_{1}}\right) \cos \left(\alpha_{j} \frac{x}{x_{1}}\right)+\sum_{j=1} C_{j} \sin \left(j \pi \frac{z}{z_{1}}\right) \cosh \left(j \pi \frac{x}{z_{1}}\right) \\
\theta_{\mathrm{III}} & =\left(\frac{\Theta_{0}}{z_{1}}+\frac{Q}{2 \lambda_{s} x_{2}}\right) z+\sum_{j=1} D_{j} \sinh \beta_{j} z \frac{\cos \left(\beta_{j}\left(x_{2}-x\right)\right)}{\cos \left(\beta_{j} x_{2}\right)}+\sum_{j=1} E_{j} \sin \left(j \pi \frac{z}{z_{1}}\right) \frac{\cosh \left(j \pi \frac{x_{2}-x}{z_{1}}\right)}{\cosh \left(j \pi \frac{x_{2}}{z_{1}}\right)}
\end{aligned}
$$

where $\alpha_{j}=\frac{(2 j-1) \pi}{2}$ and $\beta_{j}\left(x_{2}-x_{1}\right)=\frac{(2 j-1) \pi}{2}$. Hereafter, $x_{2}-x_{1}$ is simplified as $\Delta x$. The coefficients $B_{j}, D_{j}, E_{j}$ are given as follows:

$$
\begin{aligned}
B_{j} & =\frac{2(-1)^{j}}{\sinh \left(\alpha_{j} \frac{z_{1}}{x_{1}}\right)} \sum_{m=1} A_{m} \frac{\alpha_{j}}{\left(m \pi \frac{x_{1}}{x_{2}}\right)^{2}-\alpha_{j}^{2}} \cos \left(m \pi \frac{x_{1}}{x_{2}}\right), \\
D_{j} & =2(-1)^{j} \frac{x_{2}}{\Delta x} \frac{\cos \left(\beta_{j} x_{2}\right)}{\sinh \left(\beta_{j} z_{1}\right)} \sum_{m=1} A_{m} \frac{\beta_{j} x_{2}}{(m \pi)^{2}-\left(\beta_{j} x_{2}\right)^{2}} \cos \left(m \pi \frac{x_{1}}{x_{2}}\right), \\
E_{j} & =C_{j} \frac{\cosh \left(j \pi \frac{x_{1}}{z_{1}}\right) \cosh \left(j \pi \frac{x_{2}}{z_{1}}\right)}{\cosh \left(j \pi \frac{\Delta x}{z_{1}}\right)},
\end{aligned}
$$

and the other coefficients $\Theta_{0}, A_{j}$ and $C_{j}$ are obtained by solving the following $(2 n+1)$-set of simultaneous equations:

$$
\left\{\begin{array}{c}
a_{1,1} \Theta_{0}+\sum_{j=1} a_{1,2 j} A_{j}+\sum_{j=1} a_{1,2 j+1} C_{j}=0 \\
\vdots \quad a_{2 i, 2 n+1} C_{n}=0, \\
\sum_{j=1} a_{2 i, 2 j} A_{j}+\quad(i=1, \cdots, n) \\
a_{2 i+1,1} \Theta_{0}+\sum_{j=1} a_{2 i+1,2 j} A_{j}+\sum_{j=1} a_{2 i+1,2 j+1} C_{j}=\frac{Q}{2 \lambda_{s}} b_{2 i+1}, \\
\vdots
\end{array}\right.
$$


where $n$ is the number of truncation of Eq.(31) and

$$
\begin{aligned}
a_{1,1} & =\frac{x_{1}}{z_{1}} \\
a_{1,2 j} & =\sin \left(j \pi \frac{x_{1}}{x_{2}}\right)-\sum_{m=1} \frac{2}{\tanh \left(\alpha_{m} \frac{z_{1}}{x_{1}}\right)} \frac{\alpha_{m}}{\left(j \pi \frac{x_{1}}{x_{2}}\right)^{2}-\alpha_{m}^{2}} \cos \left(j \pi \frac{x_{1}}{x_{2}}\right) \\
a_{1,2 j+1} & =(-1)^{j} \sinh \left(j \pi \frac{x_{1}}{z_{1}}\right) \\
a_{2 i, 2 j} & =-2(-1)^{i} \cos \left(j \pi \frac{x_{1}}{x_{2}}\right) \sum_{m=1}\left(\frac{(i \pi) \cdot\left(\alpha_{m} \frac{z_{1}}{x_{1}}\right)}{(i \pi)^{2}+\left(\alpha_{m} \frac{z_{1}}{x_{1}}\right)^{2}} \frac{\alpha_{m}}{\left(j \pi \frac{x_{1}}{x_{2}}\right)^{2}-\alpha_{m}^{2}}\right. \\
a_{2 i, 2 n+1} & =\frac{n \pi}{2} \cosh \left(n \pi \frac{x_{1}}{z_{1}}\right)\left\{\tanh \left(n \pi \frac{x_{1}}{z_{1}}\right)+\frac{x_{f}}{\lambda_{s}} \frac{(i \pi) \cdot\left(\beta_{m} z_{1}\right)}{(i \pi)^{2}+\left(\beta_{m} z_{1}\right)^{2}} \frac{\beta_{m} x_{2}}{(j \pi)^{2}-\left(\beta_{m} x_{2}\right)^{2}}\right) \\
a_{2 i+1,1} & =\frac{\lambda_{f}}{\lambda_{s}} \frac{1}{\beta_{i} z_{1}} \\
a_{2 i+1,2 j} & \left.=-\left(j \pi+\frac{\Delta x}{z_{1}}\right)\right\} \\
a_{2 i+1,2 j+1} & =\frac{\lambda_{f}}{\lambda_{s}}(-1)^{j} j \pi \cosh \left(j \pi \frac{x_{1}}{z_{1}}\right) \frac{\beta_{i} x_{2}}{(j \pi)^{2}+\left(\beta_{i} z_{1}\right)^{2}} \\
b_{2 i+1} & =\frac{1}{x_{2} \beta_{i}}\left(1-\frac{\beta_{f} x_{2}}{\lambda_{s}}\right) \cdot
\end{aligned}
$$

From the simultaneous linear equations (33), the solution of $\Theta_{0}$ forms

$$
\Theta_{0}=\frac{Q}{2 \lambda_{s}} \frac{\mathscr{D}_{0}}{\mathscr{D}_{1}}
$$

where $\mathscr{D}_{i}(i=0,1)$ are as defined in Eqs.(25)(26).

Now, $\theta_{\mathrm{I}}$ is the temperature due to the passage of heat at the rate of $Q$ through the constriction, and subtracting the temperature difference for the passage of the same amount of heat through the solid medium when there is no constriction, $\frac{Q}{2 \lambda_{s} x_{2}}\left(z-z_{1}\right)$, the half temperature gap at the constriction $\frac{\Delta \Theta}{2}$ may be given as:

$$
\frac{\Delta \Theta}{2}=\Theta_{0}+\frac{Q}{2 \lambda_{s} x_{2}} z_{1}=\frac{Q}{2 \lambda_{s}}\left(\frac{\mathscr{D}_{0}}{\mathscr{D}_{1}}+\frac{z_{1}}{x_{2}}\right) .
$$

Therefore, the thermal resistance $R_{c}$ along the entire constriction $-z_{1} \leq z \leq z_{1}$ is

$$
R_{c}=\frac{\Delta \Theta}{Q / 2 x_{2}}=\frac{2 x_{2}}{\lambda_{s}}\left(\frac{\mathscr{D}_{0}}{\mathscr{D}_{1}}+\frac{z_{1}}{x_{2}}\right)
$$

and its equivalent length $\ell_{R}$ calculated by $\frac{\Delta \Theta}{\mathrm{d} \theta_{\mathrm{I}} /\left.\mathrm{d} z\right|_{z_{1}}}$ yields:

$$
\frac{\ell_{R}}{x_{2}}=2\left(\frac{\mathscr{D}_{0}}{\mathscr{D}_{1}}+\frac{z_{1}}{x_{2}}\right)
$$




\section{A.2 Axisymmetric case used for 3-D simulations}

The heat conduction equations of axisymmetric coordinate system are solved in the regions of I, II and III under the following boundary conditions:

$$
\begin{array}{lll}
z \geq z_{1}: & Q=\int_{0}^{r_{2}} \lambda_{s} \frac{\partial \theta_{\mathrm{I}}}{\partial z} \cdot 2 \pi r \mathrm{~d} r, \\
z=0 & : \quad \theta_{\mathrm{II}}=0 \text { and } \theta_{\mathrm{III}}=0, \\
z=z_{1}: & \theta_{\mathrm{I}}=\theta_{\mathrm{II}} \text { and } \lambda_{s} \frac{\partial \theta_{\mathrm{I}}}{\partial z}=\lambda_{s} \frac{\partial \theta_{\mathrm{II}}}{\partial z}, \quad\left(0 \leq r \leq r_{1}\right) \\
& \theta_{\mathrm{I}}=\theta_{\mathrm{III}} \text { and } \lambda_{s} \frac{\partial \theta_{\mathrm{I}}}{\partial z}=\lambda_{f} \frac{\partial \theta_{\mathrm{III}}}{\partial z}, \quad\left(r_{1} \leq r \leq r_{2}\right) \\
r=r_{1} \quad: \quad \theta_{\mathrm{II}}=\theta_{\mathrm{III}} \text { and } \lambda_{s} \frac{\partial \theta_{\mathrm{II}}}{\partial r}=\lambda_{f} \frac{\partial \theta_{\mathrm{III}}}{\partial r}, & \\
r=r_{2} \quad: \quad & \frac{\partial \theta_{\mathrm{I}}}{\partial r}=0 \text { and } \frac{\partial \theta_{\mathrm{II}}}{\partial r}=0 .
\end{array}
$$

By followingSanokawa [1968a], the general solutions for the respective regions are prescribed as:

$$
\begin{aligned}
\theta_{\mathrm{I}} & =\Theta_{0}+\frac{Q}{\lambda_{s} \pi r_{2}^{2}} z+\sum_{j=1} A_{j} \exp \left(-\gamma_{j} \frac{z-z_{1}}{r_{2}}\right) J_{0}\left(\gamma_{j} \frac{r}{r_{2}}\right), \\
\theta_{\mathrm{II}} & =\left(\frac{\Theta_{0}}{z_{1}}+\frac{Q}{\lambda_{s} \pi r_{2}^{2}}\right) z+\sum_{j=1} B_{j} \sinh \left(\alpha_{j} \frac{z}{r_{1}}\right) J_{0}\left(\alpha_{j} \frac{r}{r_{1}}\right)+\sum_{j=1} C_{j} \sin \left(j \pi \frac{z}{z_{1}}\right) I_{0}\left(j \pi \frac{r}{z_{1}}\right), \\
\theta_{\mathrm{III}} & =\left(\frac{\Theta_{0}}{z_{1}}+\frac{Q}{\lambda_{s} \pi r_{2}^{2}}\right) z+\sum_{j=1} D_{j} \sinh \left(\beta_{j} \frac{z}{r_{1}}\right) \mathrm{G}_{0}\left(\beta_{j} \frac{r}{r_{1}}\right)+\sum_{j=1} E_{j} \sin \left(j \pi \frac{z}{z_{1}}\right) \mathrm{W}_{0}\left(j \pi \frac{r}{z_{1}}\right)
\end{aligned}
$$

where $J_{n}, Y_{n}, I_{n}, K_{n}(n=0,1)$ are the regular and modified Bessel functions of the first and second kinds,

$$
\begin{aligned}
\mathrm{G}_{0}\left(\beta_{j} \frac{r}{r_{1}}\right) & \equiv J_{0}\left(\beta_{j} \frac{r}{r_{1}}\right)-\frac{J_{1}\left(\beta_{j} \frac{r_{2}}{r_{1}}\right)}{Y_{1}\left(\beta_{j} \frac{r_{2}}{r_{1}}\right)} Y_{0}\left(\beta_{j} \frac{r}{r_{1}}\right), \\
\mathrm{W}_{0}\left(j \pi \frac{r}{z_{1}}\right) & \equiv I_{0}\left(j \pi \frac{r}{z_{1}}\right)+\frac{I_{1}\left(j \pi \frac{r_{2}}{z_{1}}\right)}{K_{1}\left(j \pi \frac{r_{2}}{z_{1}}\right)} K_{0}\left(j \pi \frac{r}{z_{1}}\right),
\end{aligned}
$$

and $\alpha_{j}, \beta_{j}$ and $\gamma_{j}$ are, respectively, the roots of the following equations:

$$
J_{0}\left(\alpha_{j}\right)=0, \quad \mathrm{G}_{0}\left(\beta_{j}\right)=0, \quad J_{1}\left(\gamma_{j}\right)=0 .
$$

The coefficients $B_{j}, D_{j}, E_{j}$ are given as follows:

$$
\begin{aligned}
B_{j} & =-\sum_{m=1} A_{m} \frac{2}{\sinh \left(\alpha_{j} \frac{z_{1}}{r_{1}}\right)} \frac{\alpha_{j}}{\left(\gamma_{m} \frac{r_{1}}{r_{2}}\right)^{2}-\alpha_{j}^{2}} \frac{J_{0}\left(\gamma_{m} \frac{r_{1}}{r_{2}}\right)}{J_{1}\left(\alpha_{j}\right)}, \\
D_{j} & =\sum_{m=1} A_{m} \frac{2}{\sinh \left(\beta_{j} \frac{z_{1}}{r_{1}}\right)} \frac{1}{\left(\frac{r_{2}}{r_{1}}\right)^{2}\left(\frac{\mathrm{G}_{0}\left(\beta_{j} \frac{r_{2}}{r_{1}}\right)}{\mathrm{G}_{1}\left(\beta_{j}\right)}\right)^{2}-1} \frac{\beta_{j}}{\left(\gamma_{m} \frac{r_{1}}{r_{2}}\right)^{2}-\beta_{j}^{2}} \frac{J_{0}\left(\gamma_{m} \frac{r_{1}}{r_{2}}\right)}{\mathrm{G}_{1}\left(\beta_{j}\right)}, \\
E_{j} & =C_{j} \frac{I_{0}\left(j \pi \frac{r_{1}}{z_{1}}\right)}{\mathrm{W}_{0}\left(j \pi \frac{r_{1}}{z_{1}}\right)}
\end{aligned}
$$


where $\mathrm{G}_{1}\left(\beta_{j}\right)=J_{1}\left(\beta_{j}\right)-\frac{J_{1}\left(\beta_{j} \frac{r_{2}}{r_{1}}\right)}{Y_{1}\left(\beta_{j} \frac{r_{2}}{r_{1}}\right)} Y_{1}\left(\beta_{j}\right)$. The other coefficients $\Theta_{0}, A_{j}$ and $C_{j}$ are obtained by solving the following $(2 n+1)$-set of simultaneous linear equations:

$$
\left\{\begin{array}{c}
a_{1,1} \Theta_{0}+\sum_{j=1} a_{1,2 j} A_{j}+\sum_{j=1} a_{1,2 j+1} C_{j}=0 \\
\vdots \begin{array}{l}
\sum_{j=1} a_{2 i, 2 j} A_{j}+a_{2 i, 2 n+1} C_{n}=0, \\
a_{2 i+1,1} \Theta_{0}+\sum_{j=1} a_{2 i+1,2 j} A_{j}+\sum_{j=1} a_{2 i+1,2 j+1} C_{j}=\frac{Q}{\lambda_{s} \pi r_{2}} b_{2 i+1},
\end{array} \\
\vdots
\end{array}\right.
$$

where $n$ is the number of truncation of Eq.(36) and

$$
\begin{aligned}
& a_{1,1}=\frac{1}{2} \frac{r_{1}}{z_{1}} \\
& a_{1,2 j}=J_{1}\left(\gamma_{j} \frac{r_{1}}{r_{2}}\right)-\sum_{m=1} \frac{2}{\tanh \left(\frac{\alpha_{m}}{r_{1}} z_{1}\right)} \frac{\alpha_{m}}{\left(\gamma_{j} \frac{r_{1}}{r_{2}}\right)^{2}-\alpha_{m}^{2}} J_{0}\left(\gamma_{j} \frac{r_{1}}{r_{2}}\right) \\
& a_{1,2 j+1}=(-1)^{j} I_{1}\left(\frac{j \pi}{z_{1}} r_{1}\right) \\
& a_{2 i, 2 j}=\sum_{m=1} 2(-1)^{i} J_{0}\left(\gamma_{j} \frac{r_{1}}{r_{2}}\right)\left\{\frac{\alpha_{m}}{\left(\gamma_{j} \frac{r_{1}}{r_{2}}\right)^{2}-\alpha_{m}^{2}} \frac{\alpha_{m} \cdot i \pi \frac{r_{1}}{z_{1}}}{\alpha_{m}^{2}+\left(i \pi \frac{r_{1}}{z_{1}}\right)^{2}}\right. \\
& \left.+\frac{\lambda_{f}}{\lambda_{s}} \frac{1}{\left(\frac{r_{2}}{r_{1}}\right)^{2}\left(\frac{\mathrm{G}_{0}\left(\beta_{m} \frac{r_{2}}{r_{1}}\right)}{\mathrm{G}_{1}\left(\beta_{m}\right)}\right)^{2}-1} \frac{\beta_{m}}{\left(\gamma_{j} \frac{r_{1}}{r_{2}}\right)^{2}-\beta_{m}^{2}} \frac{\beta_{m} \cdot i \pi \frac{r_{1}}{z_{1}}}{\beta_{m}^{2}+\left(i \pi \frac{r_{1}}{z_{1}}\right)^{2}}\right\} \\
& a_{2 i, 2 n+1}=-\frac{n \pi}{2}\left\{I_{1}\left(n \pi \frac{r_{1}}{z_{1}}\right)-\frac{\lambda_{f}}{\lambda_{s}} \frac{I_{0}\left(n \pi \frac{r_{1}}{z_{1}}\right)}{\mathrm{W}_{0}\left(n \pi \frac{r_{1}}{z_{1}}\right)} \mathrm{W}_{1}\left(n \pi \frac{r_{1}}{z_{1}}\right)\right\} \\
& a_{2 i+1,1}=\frac{\lambda_{f}}{\lambda_{s}} \frac{1}{\beta_{i}} \frac{r_{1}}{z_{1}} \\
& a_{2 i+1,2 j}=-\frac{\beta_{i}}{\left(\gamma_{j} \frac{r_{1}}{r_{2}}\right)^{2}-\beta_{i}{ }^{2}} J_{0}\left(\gamma_{j} \frac{r_{1}}{r_{2}}\right)\left(\gamma_{j} \frac{r_{1}}{r_{2}}+\frac{\lambda_{f}}{\lambda_{s}} \frac{\beta_{i}}{\tanh \left(\beta_{i} \frac{z_{1}}{r_{1}}\right)}\right) \\
& a_{2 i+1,2 j+1}=\frac{\lambda_{f}}{\lambda_{s}}(-1)^{j}\left(j \pi \frac{r_{1}}{z_{1}}\right) \frac{\beta_{i}}{\beta_{i}^{2}+\left(j \pi \frac{r_{1}}{z_{1}}\right)^{2}} I_{0}\left(j \pi \frac{r_{1}}{z_{1}}\right) \\
& b_{2 i+1}=\frac{r_{1}}{r_{2}} \frac{1}{\beta_{i}}\left(1-\frac{\lambda_{f}}{\lambda_{s}}\right) \text {. }
\end{aligned}
$$

Here, $\mathrm{W}_{1}$ in Eq.(39e) originates from $\frac{\mathrm{dW}}{\mathrm{d} r}$ and defined as $\mathrm{W}_{1}\left(\frac{n \pi}{z_{1}} r_{1}\right) \equiv I_{1}\left(\frac{n \pi}{z_{1}} r_{1}\right)-\frac{I_{1}\left(\frac{n \pi}{z_{1}} r_{2}\right)}{K_{1}\left(\frac{n \pi}{z_{1}} r_{2}\right)} K_{1}\left(\frac{n \pi}{z_{1}} r_{1}\right)$. Note that Eq.(39h) is missing in the original derivation by Sanokawa [1968a]. The solution of $\Theta_{0}$ is formed as

$$
\Theta_{0}=\frac{Q}{\pi \lambda_{s} r_{2}} \frac{\mathscr{D}_{0}}{\mathscr{D}_{1}}
$$

with $\mathscr{D}_{0}$ and $\mathscr{D}_{1}$ in Eqs.(25)(26). The half temperature gap due to the presence of the constriction is estimated as:

$$
\frac{\Delta \Theta}{2}=\frac{Q}{\pi \lambda_{s} r_{2}}\left(\frac{\mathscr{D}_{0}}{\mathscr{D}_{1}}+\frac{z_{1}}{r_{2}}\right)
$$


and, therefore, the equivalent length of the thermal resistance is given as:

$$
\frac{\ell_{R}}{r_{2}}=2\left(\frac{\mathscr{D}_{0}}{\mathscr{D}_{1}}+\frac{z_{1}}{r_{2}}\right)
$$

which is apparently the identical form to the 2-D case, Eq.(35).

\section{Acknowledgements}

The authors gratefully acknowledge the financial supports of Lin Yibin Scholarship (Hangzhou, China) and Hosokawa Powder Technology Foundation (Osaka, Japan) No.J-83. This work is partly supported by Grant-in-Aid (C) No.2640111 of Japan Society for the Promotion of Science (JSPS, Japan). The authors thank the referees for his/her constructive comments and thorough inspections of the manuscript. 